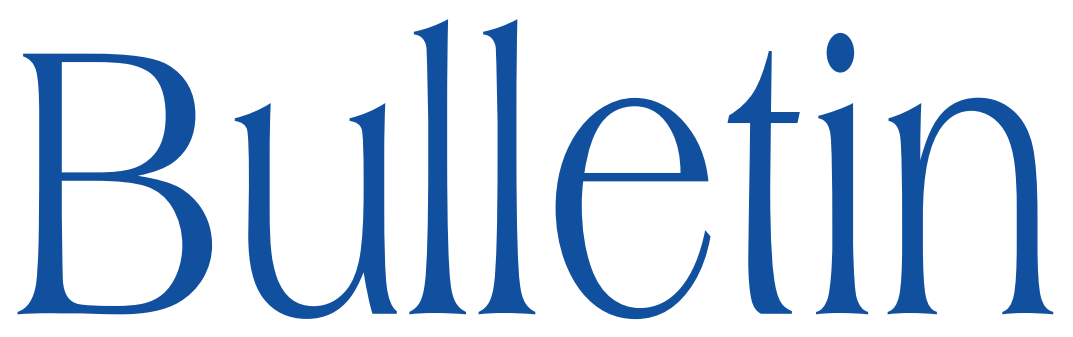

de la SOCIÉTÉ MATHÉMATIQUE DE FRANCE

\title{
VARIÉTÉS HOROSPHÉRIQUES DE FANO
}

\section{Boris Pasquier}

\section{Tome 136}

Fascicule 2

2008 


\title{
VARIÉTÉS HOROSPHÉRIQUES DE FANO
}

\author{
PAR BORIS PASQUIER
}

\begin{abstract}
Résumé. - Une variété horosphérique est une variété algébrique normale dans laquelle un groupe algébrique réductif opère avec une orbite ouverte fibrée en tores sur une variété de drapeaux. En particulier, les variétés toriques et les variétés de drapeaux sont horosphériques. Dans cet article, on classifie les variétés horosphériques de Fano en termes de certains polytopes rationnels qui généralisent les polytopes réflexifs considérés par V. Batyrev. Puis on obtient une majoration du degré des variétés horosphériques lisses de Fano, analogue à celle donnée par O. Debarre dans le cas torique. On étend un résultat récent de $\mathrm{C}$. Casagrande: les variétés horosphériques $\mathbb{Q}$-factorielles de Fano ont leur nombre de Picard majoré par deux fois la dimension.
\end{abstract}

Abstract (Fano horospherical varieties). - A horospherical variety is a normal algebraic variety where a reductive algebraic group acts with an open orbit which is a torus bundle over a flag variety. For example, toric varieties and flag varieties are horospherical. In this paper, we classify Fano horospherical varieties in terms of certain rational polytopes that generalize the reflexive polytopes considered by V. Batyrev. Then, we obtain an upper bound on the degree of smooth Fano horospherical varieties, analogus to that given by O. Debarre in the toric case. We extend a recent result of C. Casagrande: the Picard number of any Fano $\mathbb{Q}$-factorial horospherical variety is bounded by twice the dimension.

Texte reçu le 6 novembre 2006, révisé le 20 mars 2007, accepté le 4 mai 2007

Boris PASquier, UMR5582, Institut Fourier, 100 rue des Maths, BP 74, 38402 St Martin d'Hères - E-mail : Boris.Pasquier@ujf-grenoble.fr

Classification mathématique par sujets (2000). — 14I45, 14L30, 14M17, 52B20.

Mots clefs. - Variété de Fano, variété horosphérique, polytope rationnel, degré, nombre de Picard. 


\section{Introduction}

Une variété complexe projective $X$ est dite de Fano si elle est normale et si son diviseur anticanonique $-K_{X}$ est de Cartier et ample. On sait qu'il n'y a qu'un nombre fini de familles de variétés lisses, de Fano et de dimension donnée. Cependant ces familles sont seulement connues jusqu'en dimension 3.

Les variétés toriques donnent beaucoup d'exemples de variétés de Fano. Plus précisément, V. Batyrev a classifié les variétés toriques de Fano de dimension $d$ en termes des polytopes réflexifs de dimension $d[3]$ : ce sont les polytopes convexes de $\mathbb{R}^{d}$ à sommets dans $\mathbb{Z}^{d}$ contenant 0 dans leur intérieur et tels que leur polytope dual vérifie les mêmes hypothèses.

De plus, certaines propriétés ou certains invariants géométriques des variétés toriques de Fano, comme la lissité, le nombre de Picard ou le degré, se lisent facilement sur le polytope réflexif associé. Cela a permis à O. Debarre de majorer le degré $\left(-K_{X}\right)^{d}$ des variétés toriques lisses de Fano en fonction de la dimension $d$ et du nombre de Picard [12]. D'autre part, C. Casagrande a récemment donné une majoration optimale du nombre de Picard des variétés toriques $\mathbb{Q}$-factorielles et de Fano en fonction de la dimension [9].

Cet article a pour but de généraliser tous ces résultats aux variétés horosphériques. Soit $G$ un groupe algébrique réductif connexe. Un $G$-espace homogène est dit horosphérique de rang $n$ si c'est un fibré en tores $\left(\mathbb{C}^{*}\right)^{n}$ sur une variété de drapeaux.

Une variété horosphérique est un plongement d'un espace homogène horosphérique $G / H$, c'est-à-dire une $G$-variété normale contenant une orbite ouverte isomorphe à $G / H$; son rang est celui de $G / H$. Parmi les variétés horosphériques, on compte les variétés toriques (lorsque $G / H$ est un tore) et les variétés de drapeaux. Ces dernières sont lisses et de Fano.

Les variétés horosphériques font partie de la famille des variétés sphériques. Les plongements d'un espace homogène sphérique $G / H$ fixé ont été classifiés en termes d'éventails coloriés par D. Luna et T. Vust [19]. Lorsque $G / H$ est horosphérique de rang $n$, on montre que les plongements de Fano de $G / H$ sont classifiés en termes de certains polytopes rationnels, dits $G / H$-réflexifs (voir la définition 3.3). Ces polytopes sont de dimension $n$ (tout comme les éventails coloriés). Il est important de remarquer que la dimension de $G / H$ est plus grande que $n$, avec égalité si et seulement si $G / H$ est un tore; dans ce dernier cas, les polytopes $G / H$-réflexifs sont les polytopes réflexifs définis par V. Batyrev. A rang égal, les polytopes $G / H$-réflexifs peuvent être beaucoup plus nombreux que les polytopes réflexifs [22, ch.6].

V. Alexeev et M. Brion ont montré que l'ensemble des classes d'isomorphisme des variétés sphériques de Fano de dimension fixée est fini [1]. On verra que la classification précédente permet d'avoir une version effective de ce

TOME $136-2008-\mathrm{N}^{\mathrm{O}} 2$ 
résultat pour les variétés horosphériques de Fano dont l'orbite ouverte est fixée.

Dans la partie 2, on présente la classification de Luna et Vust dans le cas d'un espace homogène horosphérique $G / H$.

Dans la partie 3, on classifie les plongements de Fano de $G / H$ en termes de polytopes $G / H$-réflexifs, et on donne une borne explicite du nombre de classes d'isomorphisme de plongements de Fano de $G / H$.

Grâce à cette classification, on démontre les résultats suivants, dans la partie 4 .

THÉORÈme 1.1. - Soit $X$ une variété horosphérique de Fano, localement factorielle, de dimension $d$, de rang $n$ et de nombre de Picard $\rho$.

Si $\rho>1$ alors

$$
\left(-K_{X}\right)^{d} \leq d ! d^{d \rho+n} .
$$

Si $\rho=1$, on $a$

$$
\left(-K_{X}\right)^{d} \leq d !(d+1)^{d+n} .
$$

Une variété lisse est localement factorielle. La réciproque est vraie pour les variétés toriques mais elle est fausse pour les variétés horosphériques (un critère de lissité est donné pour les variétés horosphériques dans [22, th. 2.6] ou [25, th. 28.3]).

ThÉORÈme 1.2. - Soit $X$ une variété horosphérique de Fano, $\mathbb{Q}$-factorielle, de dimension $d$, de rang $n$ et de nombre de Picard $\rho$. On a

$$
\rho \leq n+d \leq 2 d
$$

avec $\rho=2 d$ si et seulement si $d$ est pair et $X=\left(S_{3}\right)^{d / 2}$ où $S_{3}$ est l'éclatement de $\mathbb{P}^{2}$ en trois points non alignés.

Les preuves de ces deux résultats sont inspirées de celles des résultats analogues dans le cas torique ([12], [9]). Il faut prendre en compte le fait que les polytopes $G / H$-réflexifs ne sont pas à sommets entiers comme dans le cas torique. Cependant, les sommets non entiers sont parmi un nombre fini de points rationnels qui dépendent seulement de $G / H$, ce qui permet de contrôler les changements qui apparaissent entre les cas torique et horosphérique. On utilisera alors des arguments de géométrie convexe ainsi que des éléments de combinatoire sur les groupes algébriques réductifs.

On remarquera que les variétés de nombre de Picard 1 sont souvent étudiées à part : quelles sont ces variétés? Les seules variétés toriques lisses et de nombre de Picard 1 sont les espaces projectifs. Par contre, parmi les variétés horosphériques lisses de nombre de Picard 1, on compte les variétés de drapeaux $G / P$ avec $P$ maximal, mais aussi des variétés non homogènes [23]. 
Une question naturelle se pose : est-ce que ces deux théorèmes peuvent se généraliser aux variétés sphériques?

On sait que toute variété sphérique de Fano $X$ dégénère en une variété horosphérique (ou même torique) $X_{0}$ qui est projective et $\mathbb{Q}$-Fano, c'est-à-dire qu'il existe un entier positif $k$ tel que $-k K_{X_{0}}$ est de Cartier et ample [2]. Mais l'entier $k$ peut être très grand, et la variété $X_{0}$ est en général très singulière. Ainsi tous ces résultats ne sont qu'une première étape dans la classification des variétés sphériques de Fano.

Cet article est une partie de ma thèse [22]. On y trouve notamment, dans les chapitres 6 et 7 , des exemples de polytopes $G / H$-réflexifs et une description de certaines variétés horosphériques de rang 2, lisses et de Fano.

\section{Notations}

Toutes les variétés considérées sont des variétés algébriques sur $\mathbb{C}$.

On se donne un groupe algébrique $G$ réductif (c'est-à-dire qui ne contient aucun sous-groupe distingué isomorphe à $\left.\mathbb{C}^{n}\right)$ et connexe sur $\mathbb{C}$, un sous-groupe de Borel $B$ de $G$, un tore maximal $T$ de $B$ et le radical unipotent $U$ de $B$. On note $R$ l'ensemble des racines de $(G, T), R^{+}$l'ensemble des racines positives (c'est-à-dire l'ensemble des racines de $(B, T)), S$ l'ensemble des racines simples, $\Lambda$ (respectivement $\Lambda^{+}$) le groupe des caractères de $B$ ou de $T$ (respectivement l'ensemble des caractères dominants) et $W$ le groupe de Weyl de $(G, T)$. Pour toute racine simple $\alpha$, on note $\check{\alpha}$ sa coracine et $\omega_{\alpha}$ le poids fondamental associé à $\alpha$.

Pour tout sous-groupe fermé $H$ de $G, N_{G}(H)$ désigne le normalisateur de $H$ dans $G$, et $R_{u}(H)$ est le radical unipotent de $H$.

Lorsque $I \subset S$, on note $W_{I}$ le sous-groupe de $W$ engendré par les réflexions simples $s_{\alpha}$ pour tout $\alpha \in I$, et de même $R_{I}$ (respectivement $R_{I}^{+}$) désigne l'ensemble des racines (respectivement positives) qui sont combinaisons linéaires des racines simples de $I$. On note $P_{I}$ le sous-groupe parabolique de $G$ engendré par $B$ et $W_{I}$. Alors $I \longmapsto P_{I}$ est une bijection entre l'ensemble des parties de $S$ et l'ensemble des sous-groupes paraboliques contenant $B$ [24, th. 8.4.3].

Pour tout caractère dominant $\lambda$, on note $V(\lambda)$ le $G$-module simple de plus grand poids $\lambda[16, \mathrm{ch} . \mathrm{XI}]$. On désigne par $v_{\lambda}$ un vecteur propre de $V(\lambda)$ de poids $\lambda$, et le stabilisateur de la droite $\mathbb{C} v_{\lambda}$ est un sous-groupe parabolique de $G$ contenant $B$ qu'on note $P(\lambda)$. En écrivant $\lambda=\sum_{\alpha \in S} x_{\alpha} \omega_{\alpha}$, les $x_{\alpha}$ étant des entiers positifs ou nuls, on a $P(\lambda)=P_{I}$ où $I$ est l'ensemble des racines simples $\alpha$ telles que $x_{\alpha}$ soit nul. 
Les $G$-modules considérés seront toujours rationnels et de dimension finie. Si $V$ est un $G$-module, on note $V^{U}$ l'ensemble des points fixes de $V$ sous l'action de $U$ et $V^{(B)}$ l'ensemble des vecteurs propres de $V$ sous l'action de $B$. Comme $V$ est semi-simple, on a une décomposition en $G$-modules simples (où les $m_{\lambda}$ sont des entiers positifs ou nuls) :

$$
V=\bigoplus_{\lambda \in \Lambda^{+}} V(\lambda) \bigoplus m_{\lambda} \quad \text { et } \quad V^{U}=\bigoplus_{\lambda \in \Lambda^{+}}\left(\mathbb{C} v_{\lambda}\right) \bigoplus^{m_{\lambda}} .
$$

DÉfinition 2.1. - Un sous-groupe fermé $H$ de $G$ contenant $U$ est dit horosphérique. Dans ce cas, on dit aussi que l'espace homogène $G / H$ est horosphérique.

EXEMPle 2.1. - Soit $P$ un sous-groupe parabolique de $G$ contenant $B$ et soient $\chi_{1}, \ldots, \chi_{n}$ des caractères de $P$. Alors l'intersection des noyaux des $\chi_{i}$ dans $P$ est un sous-groupe horosphérique.

En fait tout sous-groupe horosphérique $H$ est de cette forme.

Proposition 2.2. - Soit $H$ un sous-groupe horosphérique de $G$. Il existe un unique sous-groupe parabolique $P$ contenant $B$ tel que $H$ soit l'intersection de noyaux de caractères de $P$. De plus, $P=N_{G}(H)$.

Démonstration. - D'après le théorème de Chevalley [16, 11.2], il existe un $G$ module $V$ et une droite $L$ de $V$ telles que $H$ soit le stabilisateur de $L$, c'est-à-dire $H=\{x \in G \mid x . L=L\}$.

Décomposons $V$ en somme directe de $G$-modules simples :

$$
V=\bigoplus_{\lambda \in \Lambda_{0}^{+}} V(\lambda)^{\oplus m_{\lambda}}
$$

avec $m_{\lambda}$ non nul pour tout $\lambda$ dans un sous-ensemble fini $\Lambda_{0}^{+}$de $\Lambda^{+}$. Comme $U \subset H$, on a $L \subset V^{U}=\oplus_{\lambda \in \Lambda_{0}^{+}}\left(\mathbb{C} v_{\lambda}\right)^{\oplus m_{\lambda}}$. Soit $\Lambda_{1}^{+} \subset \Lambda_{0}^{+}$un sous-ensemble minimal tel que $L \subset \oplus_{\lambda \in \Lambda_{1}^{+}}\left(\mathbb{C} v_{\lambda}\right)^{\oplus m_{\lambda}}$. Alors il existe $V^{\prime}=\oplus_{\lambda \in \Lambda_{1}^{+}} V(\lambda) \subset V$ tel que la projection $L^{\prime}$ de $L$ sur $V^{\prime}$ vérifie $H=\left\{x \in G \mid x \cdot L^{\prime}=L^{\prime}\right\}$. On peut donc supposer que $L$ est engendrée par un vecteur de la forme $\sum_{\lambda \in \Lambda_{1}^{+}} a_{\lambda} v_{\lambda}$ avec tous les $a_{\lambda} \neq 0$. Notons $P=\bigcap_{\lambda \in \Lambda_{1}^{+}} P(\lambda)$.

Montrons que $P$ convient. Le théorème de Chevalley nous dit que

$$
\begin{aligned}
H & =\left\{x \in G \mid \exists \lambda_{0}(x) \in \mathbb{C}^{*}, x . v=\lambda_{0}(x) v\right\} \\
& =\left\{x \in \bigcap_{\lambda \in \Lambda_{1}^{+}} P(\lambda) \mid \forall \lambda, \mu \in \Lambda_{1}^{+}, \lambda(x)=\mu(x)\right\} .
\end{aligned}
$$

Soit $\mu \in \Lambda_{1}^{+}$, alors $H=\bigcap_{\lambda \in \Lambda_{1}^{+}} \operatorname{ker}(\lambda-\mu) \subset P$. 
Montrons que $P=N_{G}(H)$. On a clairement $P \subset N_{G}(H)$, de plus $R_{u}(H)=$ $R_{u}(P)$ et $P=N_{G}\left(R_{u}(P)\right)$, donc $N_{G}(H) \subset N_{G}\left(R_{u}(H)\right)=P$.

DÉfinition 2.3. - Soit $H$ un sous-groupe horosphérique. On note $I$ le sousensemble de $S$ tel que $P=P_{I}$. Puis on définit $M$ comme l'ensemble des caractères de $P$ dont la restriction à $H$ est triviale; c'est un sous-réseau de $\Lambda$. On note $N$ le réseau dual de $M$. Le rang de $M$ est appelé le rang de $G / H$; on le note $n$. On notera aussi $d$ la dimension de $G / H$, on a évidemment

$$
d=n+\operatorname{dim}(G / P)=n+\sharp\left(R^{+} \backslash R_{I}^{+}\right) .
$$

On pose $M_{\mathbb{R}}:=M \otimes_{\mathbb{Z}} \mathbb{R}$ et $N_{\mathbb{R}}:=N \otimes_{\mathbb{Z}} \mathbb{R}$.

Remarque 2.2. - L'espace homogène $G / H$ est l'espace total d'une fibration sur la variété de drapeaux $G / P$ de fibre le tore $P / H$. Ce dernier est isomorphe au tore dual de $M$ par l'application :

$$
\begin{gathered}
P / H \longrightarrow\left\{\text { homomorphismes de groupes } M \rightarrow \mathbb{C}^{*}\right\} \\
p H \longmapsto \chi(p)] .
\end{gathered}
$$

On va classifier les espaces homogènes horosphériques $G / H$ en termes de sous-réseaux de $\Lambda$ et de sous-ensembles de $S$.

Proposition 2.4. - La constuction ci-dessus qui à un espace homogène horosphérique $G / H$ associe le couple $(M, I)$ définit une bijection de l'ensemble des $G$-espaces homogènes horosphériques sur l'ensemble des couples $(M, I)$, où $I$ est un sous-ensemble de $S$ et $M$ un sous-réseau de $\Lambda$ tel que pour tout $\alpha \in I$ et $\chi \in M,\langle\chi, \check{\alpha}\rangle=0$.

Démonstration. - Remarquons d'abord que pour tout $\chi \in \Lambda$, la condition $\langle\chi, \check{\alpha}\rangle=0$ pour tout $\alpha \in I$ est équivalente au fait que $\chi$ s'étend en un caractère de $P_{I}$.

Ensuite, à un couple $(M, I)$ vérifiant cette condition, on associe l'espace homogène horosphérique $G / H$, où $H$ est l'intersection des noyaux des caractères $\chi \in M$ dans $P_{I}$. On vérifie alors facilement que les deux applications sont inverses l'une de l'autre.

DÉFInition 2.5. - Un plongement d'un espace homogène $G / H$ est un couple $(X, x)$, où $X$ est une $G$-variété algébrique normale et $x$ est un point de $X$, tels que l'orbite de $x$ dans $X$ soit ouverte et isomorphe à $G / H$.

Deux plongements $(X, x)$ et $\left(X^{\prime}, x^{\prime}\right)$ sont isomorphes s'il existe un isomorphisme $G$-équivariant de $X$ sur $X^{\prime}$ qui envoie $x$ sur $x^{\prime}$.

Une variété horosphérique est une $G$-variété algébrique normale qui contient une orbite ouverte isomorphe à un espace homogène horosphérique. Le rang d'une variété horosphérique est le rang de sa $G$-orbite ouverte.

TOME $136-2008-\mathrm{N}^{\mathrm{O}} 2$ 
Un espace homogène $G / H$ est dit sphérique s'il contient une orbite ouverte sous l'action d'un sous-groupe de Borel de $G$. Une variété sphérique est une $G$-variété algébrique normale qui contient une orbite ouverte isomorphe à un espace homogène sphérique. Toute variété horosphérique est sphérique; ceci résulte en effet de la décomposition de Bruhat.

Dans la suite de l'article, le point d'un plongement est sous-entendu : « soit $X$ un plongement de $G / H$ » signifie rigoureusement « soit $(X, x)$ un plongement de $G / H \gg$.

La classification des plongements d'un espace homogène sphérique fixé est obtenue par l'étude de leurs orbites sous les actions de $G$ et $B$, mais aussi de leurs diviseurs irréductibles stables sous ces actions.

DÉFInItion 2.6. - Soit $G / H$ un espace homogène sphérique. On note $\mathcal{D}$ l'ensemble des diviseurs irréductibles de $G / H$ qui sont stables par $B$ mais non par $G$. Les éléments de $\mathcal{D}$ sont appelés couleurs.

Soit $X$ un plongement de $G / H$. On note $X_{1}, \ldots, X_{m}$ les diviseurs irréductibles de $X$ stables par $G$. On peut identifier $\mathcal{D}$ avec l'ensemble des diviseurs irréductibles de $X$ qui sont stables par $B$ mais non par $G$. Ainsi, $\mathcal{D} \cup\left\{X_{1}, \ldots, X_{m}\right\}$ est l'ensemble des diviseurs irréductibles $B$-stables de $X$.

Une couleur de $X$ est une couleur qui contient une $G$-orbite fermée.

Si $G / H$ est horosphérique, l'ensemble des $B$-orbites de codimension 1 de $G / H$ est l'ensemble des $B w_{0} s_{\alpha} P / H$ lorsque $\alpha$ décrit $S \backslash I$ et où $w_{0}$ est l'élément de longueur maximale dans $W$. Les couleurs sont alors les adhérences $D_{\alpha}$ des $B$-orbites $B w_{0} s_{\alpha} P / H$ dans $G / H$ et $\mathcal{D}$ est en bijection avec $S \backslash I$.

DÉFInition 2.7. - Une variété sphérique est simple si elle ne contient qu'une seule orbite fermée. Si $G / H$ est un espace homogène sphérique, alors tout plongement de $G / H$ est recouvert par les plongements simples de $G / H$ qu'il contient.

Une variété sphérique est toroïdale si elle n'a aucune couleur.

Soit $X$ un plongement d'un espace homogène horosphérique $G / H$. Définissons une application

$$
\sigma: \mathcal{D} \cup\left\{X_{1}, \ldots, X_{m}\right\} \longrightarrow N
$$

de la façon suivante ${ }^{(1)}$. Soit $D$ un diviseur $B$-stable de $X$. Il définit naturellement une valuation $v_{D}, B$-invariante, du corps des fonctions rationnelles $\mathbb{C}(G / H)=\mathbb{C}(X)$. On en déduit donc un homomorphisme de groupes $\mathbb{C}(G / H)^{(B)} / \mathbb{C}^{*} \longrightarrow \mathbb{Z}$. En remarquant ensuite que $M$ est isomorphe à

(1) Elle est définie de la même façon dans le cas sphérique, où on pose $M=\mathbb{C}(G / H)^{(B)} / \mathbb{C}^{*}$ (pour plus de détails, se référer à [17]). 
$\mathbb{C}(G / H)^{(B)} / \mathbb{C}^{*}$, la restriction de $v_{D}$ à $\mathbb{C}(G / H)^{(B)} / \mathbb{C}^{*}$ définit alors un élément de $N$ qu'on note $\sigma(D)$.

Notons que la restriction de $\sigma$ à $\mathcal{D}$ ne dépend pas de $X$ mais que de $G / H$. En fait, si $\alpha \in S \backslash I$, l'image par $\sigma$ de la couleur $D_{\alpha}$ est simplement la restriction à $M$ de $\check{\alpha}$. Dans ce cas, on notera cette image $\check{\alpha}_{M}$ au lieu de $\sigma\left(D_{\alpha}\right)$. Cependant, la restriction de $\sigma$ à $\mathcal{D}$ peut ne pas être injective.

Exemple 2.3. - Quand $G / H$ est horosphérique, on rappelle que $P / H$ est isomorphe au tore dual de $M$ par l'application $p \in P \mapsto(\chi \in M \mapsto \chi(p))$. Soit $Y$ une variété torique sous l'action de ce tore; $P$ agit alors sur $Y$.

Soit $G \times{ }^{P} Y$ le quotient de $G \times Y$ par la relation d'équivalence $(g, y) \sim$ $\left(g p^{-1}, p . y\right)$ pour tout $g \in G, p \in P$ et $y \in Y$. Alors $X=G \times{ }^{P} Y$ est une variété algébrique normale munie d'une fibration $G \times{ }^{P} Y \longrightarrow G / P$. C'est aussi un plongement de $G / H$ et les diviseurs $X_{i}$ sont les $G \times{ }^{P} Y_{i}$ où les $Y_{i}$ sontles diviseurs irréductibles de $Y$ stables par le tore. Et pour tout $\alpha$ dans $S \backslash I, D_{\alpha}$ est $\overline{B w_{0} s_{\alpha} P} \times{ }^{P} Y$. On remarque alors que chaque couleur ne contient aucune $G$-orbite de $X$ : c'est une variété horosphérique toroïdale. En fait, les variétés horosphériques toroïdales sont toujours de la forme ci-dessus, c'est-à-dire des fibrés sur une variété de drapeaux, de fibre une variété torique (cela résulte du théorème 2.5 et de l'exemple 2.6).

Lorsqu'on se donne un espace homogène horosphérique $G / H$ de rang $n$, on lui associe un sous-groupe parabolique $P$, un ensemble $I \subset S$ et un réseau $N \subset N_{\mathbb{R}}$ de rang $n$ (voir la proposition 2.2 et la définition 2.3). On a aussi l'ensemble des couleurs $\mathcal{D}$ (voir la définition 2.6), et l'application $\sigma: \mathcal{D} \longrightarrow N$ (2.2.1).

DÉFInition 2.8. - Soit $G / H$ un espace homogène horosphérique ${ }^{(2)}$ fixé (avec toutes les données associées ci-dessus).

Un cône colorié de $N_{\mathbb{R}}$ est un couple $(\mathcal{C}, \mathcal{F})$ où $\mathcal{C}$ est un cône convexe de $N_{\mathbb{R}}$ et $\mathcal{F}$ est un sous-ensemble de $\mathcal{D}$ appelé l'ensemble des couleurs du cône colorié, tel que

(i) $\mathcal{C}$ est engendré par un nombre fini d'éléments du réseau $N$ et contient $\sigma(\mathcal{F})$

(ii) $\mathcal{C}$ est saillant (c'est-à-dire ne contient aucune droite) et $\sigma(\mathcal{F})$ ne contient pas l'origine.

(2) Si $G / H$ est sphérique, la définition des cônes et éventails coloriés est quasiment identique (voir [17, chap. 4]).

TOME $136-2008-\mathrm{N}^{\mathrm{O}} 2$ 
Une face coloriée d'un cône colorié $(\mathcal{C}, \mathcal{F})$ est un couple $\left(\mathcal{C}^{\prime}, \mathcal{F}^{\prime}\right)$ où $\mathcal{C}^{\prime}$ est une face du cône $\mathcal{C}$ et $\mathcal{F}^{\prime}$ est l'ensemble des éléments de $\mathcal{F}$ dont l'image par $\sigma$ est dans $\mathcal{C}^{\prime}$.

Un éventail colorié de $N_{\mathbb{R}}$ est un ensemble fini $\mathbb{F}$ de cônes coloriés tel que

(i) toute face coloriée d'un cône colorié de $\mathbb{F}$ est dans $\mathbb{F}$,

(ii) pour tout élément $u$ de $N_{\mathbb{R}}$, il existe au plus un cône colorié $(\mathcal{C}, \mathcal{F})$ de $\mathbb{F}$ tel que $u$ soit dans l'intérieur relatif de $\mathcal{C}$.

Un éventail colorié $\mathbb{F}$ est dit complet si pour tout élément $x$ de $N_{\mathbb{R}}$, il existe un cône colorié $(\mathcal{C}, \mathcal{F})$ de $\mathbb{F}$ tel que $x$ soit dans $\mathcal{C}$.

On dira qu'un élément $D$ de $\mathcal{D}$ est une couleur de $\mathbb{F}$ s'il existe un cône colorié de $\mathbb{F}$ dont $D$ est une couleur.

REMARQUes 2.4. - Lorsque $G$ est un tore, l'ensemble des couleurs $\mathcal{D}$ est vide, et on retrouve la définition d'un éventail.

La dernière condition dans la définition d'un éventail colorié implique que l'intersection de deux cônes coloriés est une face coloriée commune.

Si deux couleurs ont la même image par $\sigma$, alors il se peut qu'un cône colorié ne possède qu'une des deux couleurs.

On obtient un cône colorié $(\mathcal{C}, \mathcal{F})$ à partir d'un plongement simple $X$ de la façon suivante. Soit $Y$ l'unique $G$-orbite fermée de $X$; alors $\mathcal{F}$ est l'ensemble des couleurs qui contiennent $Y$. Ensuite $\mathcal{C}$ est le cône engendré par $\sigma(\mathcal{F})$ et l'ensemble des $\sigma(D)$ lorsque $D$ parcourt l'ensemble des diviseurs irréductibles de $X$ stables par $G$.

Pour un plongement quelconque $X$, l'éventail colorié associé est l'ensemble des cônes coloriés associés aux plongements simples inclus dans $X$. On remarque que les couleurs de $X$ sont les mêmes que les couleurs de l'éventail colorié associé à $X$.

ThÉORÈme 2.5 (cas particulier ${ }^{(4)}$ du théorème 4.3 de [17])

Soit $G / H$ un espace homogène horosphérique. La constuction ci-dessus définit une bijection entre l'ensemble des classes d'isomorphisme de plongements de $G / H$ (définition 2.5) et l'ensemble des éventails coloriés de $N_{\mathbb{R}}$. De plus, les plongements complets correspondent aux éventails coloriés complets.

Exemple 2.6. - Soit $X=G \times{ }^{P} Y$ un plongement toroïdal de $G / H$ comme dans l'exemple 2.3. Notons $\mathbb{E}$ l'éventail de $Y$ dans $N_{\mathbb{R}}$; alors l'éventail colorié associé à $X$ est l'ensemble des cônes coloriés $(\mathcal{C}, \varnothing)$ lorsque $\mathcal{C}$ parcourt $\mathbb{E}$.

(4) L'énoncé du théorème de D. Luna et Th. Vust [17, th.4.3] est le même que celui du théorème ci-dessus avec $G / H$ sphérique. 


\section{Classification des plongements de Fano}

Le but de cette partie est de classifier, en termes de polytopes, les plongements projectifs d'un espace homogène horosphérique fixé qui sont de Fano, de façon à généraliser la classification des variétés toriques de Fano.

Rappelons qu'une variété projective est de Fano si elle est normale et si son diviseur anticanonique est de Cartier et ample. Dans cette partie, on fixe un espace homogène horosphérique $G / H$. Les notations sont celles de la partie 2 .

Lorsque $G / H$ est de rang 0 , c'est-à-dire lorsque $H$ est un sous-groupe parabolique $P$, le seul plongement est la variété de drapeaux $G / P$, qui est lisse et de Fano. Je me place maintenant dans le cas où $G / H$ est de rang au moins 1 . Les éventails coloriés complets considérés ne seront donc pas réduits au point 0 .

La première étape consiste à déterminer un diviseur anticanonique des plongements de $G / H$.

Proposition 3.1. - Soit $X$ un plongement projectif de $G / H$. Un diviseur anticanonique de $X$ est

$$
-K_{X}=\sum_{i=1}^{m} X_{i}+\sum_{\alpha \in S \backslash I} a_{\alpha} D_{\alpha}
$$

où $a_{\alpha}$ est l'entier $\left\langle 2 \rho^{P}, \check{\alpha}\right\rangle$ et $2 \rho^{P}$ est le caractère donné par $\sum_{\alpha \in R^{+} \backslash R_{I}} \alpha$.

Pour prouver ce résultat (qui est un corollaire de $[8$, th.4.2]), on se ramène au cas où $X$ est un plongement toroïdal. On note $\pi: X \longrightarrow G / P$ la fibration de fibre torique $Y$. On a alors $K_{X}=\pi^{*}\left(K_{G / P}\right)+K_{\pi}$. La fin de la preuve utilise le résultat connu dans le cas torique $-K_{X}=\sum_{i=1}^{m} X_{i}$ et le résultat pour les variétés de drapeaux

$$
-K_{G / P}=\sum_{\alpha \in S \backslash I} a_{\alpha} D_{\alpha} .
$$

Par construction de l'éventail colorié associé à $X$, les diviseurs irréductibles $G$-stables $X_{i}$ correspondent aux arêtes de l'éventail $\mathbb{F}$ associé à $X$ qui ne sont pas engendrées par une couleur. On note $x_{i} \in N$ l'élément primitif de l'arête correspondante à $X_{i}$.

Afin de déterminer les plongements de Fano de $G / H$, on utilise la caractérisation suivante des diviseurs amples [7, th.3.3].

Proposition 3.2. - Soient $X$ un plongement projectif de $G / H, \mathbb{F}$ son éventail colorié et $D$ un diviseur de Weil de la forme

$$
\sum_{i=1}^{m} b_{i} X_{i}+\sum_{\alpha \in S \backslash I} b_{\alpha} D_{\alpha}
$$

TOME $136-2008-\mathrm{N}^{\mathrm{O}} 2$ 
où les $b_{i}$ et les $b_{\alpha}$ sont dans $\mathbb{Z}$.

Alors $D$ est de Cartier si et seulement si pour tout cône colorié $(\mathcal{C}, \mathcal{F})$ de $\mathbb{F}$, il existe $\chi_{\mathcal{C}}$ dans $M$ tel que

$$
\begin{gathered}
\forall x_{i} \in \mathcal{C},\left\langle x_{i}, \chi_{C}\right\rangle=b_{i} \\
\text { et } \forall D_{\alpha} \in \mathcal{F},\left\langle\check{\alpha}_{M}, \chi_{\mathcal{C}}\right\rangle=b_{\alpha} .
\end{gathered}
$$

Lorsque $D$ est de Cartier on peut alors définir une application $h_{D}$ de $N_{\mathbb{R}}$ dans $\mathbb{R}$, comme suit. Soit $x$ un élément de $N_{\mathbb{R}}$. Comme $X$ est projectif, $\mathbb{F}$ est complet donc il existe un unique cône colorié maximal $(\mathcal{C}, \mathcal{F})$ de $\mathbb{F}$ tel que $x$ soit dans $\mathcal{C}$, et on pose alors $h_{D}(x)=\left\langle x, \chi_{\mathcal{C}}\right\rangle$. C'est une application linéaire sur chaque cône.

Le diviseur (de Cartier) D est ample si et seulement si :

(i) l'application $h_{D}$ est strictement convexe (c'est-à-dire, pour chaque cône maximal $\mathcal{C}$, l'application linéaire $\chi_{\mathcal{C}}$ est strictement supérieure sur $\mathcal{C}$ aux applications linéaires $\chi_{\mathcal{C}^{\prime}}$, pour tout cône maximal $\mathcal{C}^{\prime}$ distinct de $\left.\mathcal{C}\right)$;

(ii) pour tout cône colorié $(\mathcal{C}, \mathcal{F})$ de $\mathbb{F}$ et pour tout $\alpha$ tel que $D_{\alpha}$ ne soit pas dans $\mathcal{F}$, on a

$$
\left\langle\check{\alpha}_{M}, \chi_{\mathcal{C}}\right\rangle\left\langle b_{\alpha} .\right.
$$

On définit $\mathcal{D}_{X}$ comme l'ensemble des $\alpha \in S \backslash I$ tels que $D_{\alpha}$ soit une couleur de $X$.

Lorsque $D=-K_{X}$ est ample, l'application $h_{D}$ vérifie $h_{D}\left(x_{1}\right)=\cdots=$ $h_{D}\left(x_{m}\right)=1$ pour tout $i \in\{1, \ldots, m\}$, et $h_{D}\left(\check{\alpha}_{M}\right)=a_{\alpha}$ pour tout $\alpha \in \mathcal{D}_{X}$. L'ensemble

$$
Q=\left\{u \in N_{\mathbb{R}} \mid \forall \mathcal{C} \in \mathbb{F},\left\langle\chi_{\mathcal{C}}, u\right\rangle \leq 1\right\}
$$

est alors un polytope convexe contenant 0 dans son intérieur et dont les sommets sont $x_{1}, \ldots, x_{m}$ et certains des $\frac{\check{\alpha}_{M}}{a_{\alpha}}$ où $\alpha \in \mathcal{D}_{X}$. De plus les points $\frac{\check{\alpha}_{M}}{a_{\alpha}}$ où $\alpha \notin \mathcal{D}_{X}$ sont dans l'intérieur de $Q$, et les $\frac{\check{\alpha}_{M}}{a_{\alpha}}$ où $\alpha \in \mathcal{D}_{X}$ sont dans le bord de $Q$. En particulier, $Q$ est un polytope convexe rationnel.

On définit le polytope dual de $Q$ par

$$
Q^{*}=\left\{v \in M_{\mathbb{R}} \mid \forall u \in Q,\langle v, u\rangle \geq-1\right\} .
$$

Les sommets de $Q^{*}$ sont en bijection avec les faces maximales de $Q$. Ainsi $Q^{*}$ est l'enveloppe convexe des $-\chi_{\mathcal{C}}$ où $\mathcal{C}$ décrit l'ensemble des cônes coloriés maximaux de $\mathbb{F}$. En particulier, $Q^{*}$ est à sommets dans $M$.

Je regroupe toutes ces propriétés dans la définition suivante.

DÉFInition 3.3. - Soit $G / H$ un espace homogène horosphérique. Un polytope convexe $Q$ de $N_{\mathbb{R}}$ est dit $G / H$-réflexif si les trois conditions suivantes sont vérifiées : 
(1) $Q$ est à sommets dans $N \cup\left\{\frac{\check{\check{\alpha}}_{M}}{a_{\alpha}} \mid \alpha \in S \backslash I\right\}$, et contient 0 dans son intérieur.

(2) $Q^{*}$ est à sommets dans $M$.

(3) Pour tout $\alpha \in S \backslash I, \frac{\check{\alpha}_{M}}{a_{\alpha}} \in Q$.

EXemples 3.1. - Dans les figures, on représente les $\frac{\check{\alpha}_{M}}{a_{\alpha}}$ par des points blancs et l'origine par un point noir. Les exemples ci-dessous sont tels qu'un point blanc est associé à une seule couleur. Mais il faut tout de même noter qu'il peut y avoir des cas où un même point $\frac{\check{\alpha}_{M}}{a_{\alpha}}$ correspond à plusieurs couleurs.

(1) Dans le cas $G=S L_{2}$ et $H=U=\left\{\left(\begin{array}{ll}1 & * \\ 0 & 1\end{array}\right)\right\}$, on n'a qu'une couleur et $a_{\alpha}=2$. Les seuls polytopes $S L_{2} / U$-réflexifs sont les segments suivants.

Et leurs duaux sont respectivement :

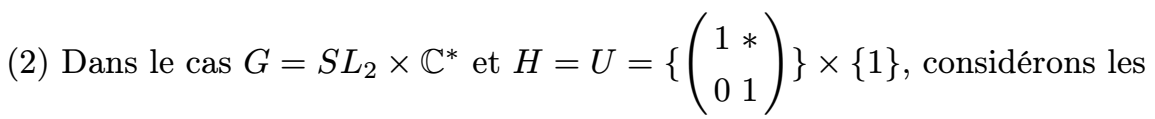
trois polytopes suivants. Les deux premiers sont $G / H$-réflexifs mais le troisième ne l'est pas car son dual n'est pas à sommets entiers.

Les polytopes duaux sont respectivement :
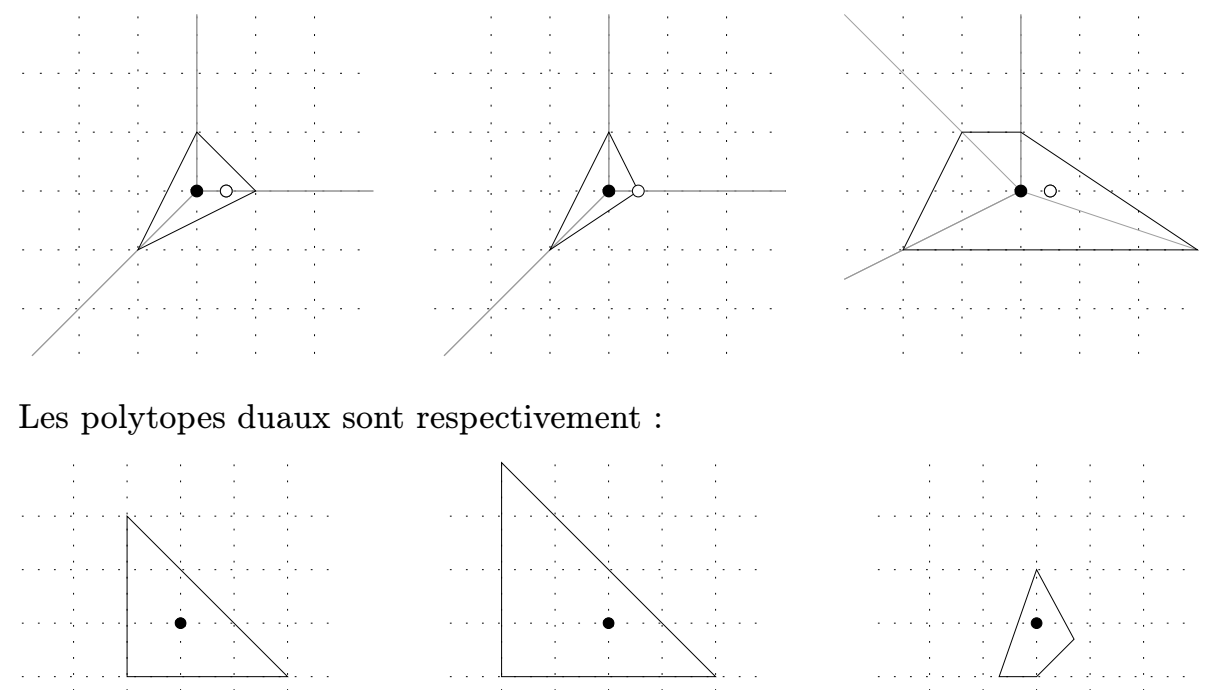
REMARque 3.2. - La condition (3) est équivalente à la condition $\left(3^{\prime}\right): 2 \rho^{P}+Q^{*}$ (le translaté de $Q^{*}$ par $2 \rho^{P}$ dans $\Lambda_{\mathbb{R}}$ ) est inclus dans $\Lambda_{\mathbb{R}}^{+}$.

En fait, $2 \rho^{P}+Q^{*}$ est le polytope moment de $\left(X,-K_{X}\right)$ [7], c'est-à-dire

$$
H^{0}\left(X,-K_{X}\right)=\bigoplus_{\lambda \in Q^{*}} V\left(2 \rho^{P}+\lambda\right) .
$$

En effet, si $s$ est la section canonique de $-K_{X}$, on a l'isomorphisme suivant

$$
\begin{aligned}
\left\{f \in \mathbb{C}(G / H)^{(B)} \mid \operatorname{div}(f)-K_{X} \geq 0\right\} & \longrightarrow H^{0}\left(X,-K_{X}\right)^{(B)} \\
f & \longmapsto f s .
\end{aligned}
$$

De plus $s$ est de poids $2 \rho^{P}=\sum_{\alpha \in S \backslash I} a_{\alpha} \omega_{\alpha}$ sous l'action de $B$, et si $f \in$ $\mathbb{C}(G / H)^{(B)}$ est de poids $\chi$ alors

$$
\operatorname{div}(f)-K_{X}=\sum_{i=1}^{m}\left(1+\left\langle\chi, x_{i}\right\rangle\right) X_{i}+\sum_{\alpha \in S \backslash I}\left(a_{\alpha}+\left\langle\chi, \check{\alpha}_{M}\right\rangle\right) D_{\alpha} .
$$

Donc les poids de $H^{0}\left(X,-K_{X}\right)^{(B)}$ sont bien les caractères qui sont dans le polytope $2 \rho^{P}+Q^{*}$.

Il peut être intéressant aussi de regarder les cas où $-K_{X}$ est seulement $\mathbb{Q}$ Cartier (c'est-à-dire un multiple de $-K_{X}$ est de Cartier) et ample : on dira alors que $X$ est $\mathbb{Q}$-Fano. Dans ce cas, $Q$ ne vérifie plus la condition (2). On dit alors qu'un polytope convexe de $N_{\mathbb{R}}$ est $\mathbb{Q}$ - $G / H$-réflexif s'il vérifie la condition (3) de la définition 3.3 et la condition

$\left(1^{\prime}\right)$ les sommets de $Q$ sont des éléments primitifs de $N$ ou des éléments de $\left\{\frac{\check{\alpha}_{M}}{a_{\alpha}}, \alpha \in S \backslash I\right\}$, et $Q$ contient 0 dans son intérieur.

On peut remarquer qu'un polytope $G / H$-réflexif vérifie aussi la condition $\left(1^{\prime}\right)$ car (1) et (2) impliquent $\left(1^{\prime}\right)$.

Le dernier polytope de l'exemple 3.1 (2) est $\mathbb{Q}-G / H$-réflexif.

Remarquons que dans le cas torique, la définition d'un polytope $\left(\mathbb{C}^{*}\right)^{n}$ réflexif est celle d'un polytope réflexif donnée par V. Batyrev. L'ensemble des classes d'isomorphisme des variétés toriques de Fano de dimension $n$ est en bijection avec l'ensemble des polytopes réflexifs de $\mathbb{Z}^{n}[3]$. La proposition suivante généralise alors cette classification aux variétés horosphériques de Fano.

Proposition 3.4. - Soit $G / H$ un espace homogène horosphérique. L'application qui à un plongement $X$ de Fano de $G / H$ associe le polytope $Q$ défini en (3.0.1) est une bijection de l'ensemble des plongements de Fano de $G / H$ (respectivement $\mathbb{Q}$-Fano) à isomorphisme près, sur l'ensemble des polytopes $G / H$ réflexifs (respectivement $\mathbb{Q}-G / H$-réflexifs) de $N_{\mathbb{R}}$. 
Démonstration. - Il suffit de définir l'application inverse. Soit $Q$ un polytope $G / H$-réflexif de $N_{\mathbb{R}}$; on lui associe alors le plongement $X(Q)$ de $G / H$ dont l'éventail colorié est l'ensemble des cônes coloriés $(\mathcal{C}, \mathcal{F})$ (et leurs faces coloriées) tels que $\mathcal{C}$ soit le cône engendré par une face maximale $F$ de $Q$, et $\mathcal{F}$ soit l'ensemble des $D_{\alpha}$ vérifiant $\frac{\check{\alpha}_{M}}{a_{\alpha}} \in F$.

Alors on vérifie que $-K_{X(Q)}$ est de Cartier $\left(-\chi_{\mathcal{C}}\right.$ est le sommet de $Q^{*}$ associé à $F$ ) et ample, par convexité de $Q$ et par la condition (3) de la définition 3.3. Ensuite, le polytope associé à $X(Q)$ (3.0.1) est bien $Q$ par construction.

De même, si $X$ est un plongement de Fano et $Q$ est le polytope $G / H$-réflexif associé, on a $X(Q)=X$.

Lorsque $Q$ est un polytope $\mathbb{Q}-G / H$-réflexif, on construit $X(Q)$ exactement de la même façon, et on vérifie aussi que cette application est l'inverse de l'application $X \mapsto Q(X)$.

REMARQUe 3.3. - Les couleurs d'un plongement $X$ de Fano sont les couleurs $D_{\alpha}$ telles que $\frac{\check{\alpha}_{M}}{a_{\alpha}}$ se trouve sur le bord du polytope $G / H$-réflexif associé à $X$. Supposons que pour $\alpha \neq \beta$ on ait $\frac{\check{\alpha}_{M}}{a_{\alpha}}=\frac{\check{\beta}_{M}}{a_{\beta}}$. Soit $X$ un plongement de Fano de $G / H$. Alors si $\frac{\check{\alpha}_{M}}{a_{\alpha}}$ est sur le bord du polytope $G / H$-réflexif associé à $X$ alors $\frac{\breve{\beta}_{M}}{a_{\beta}}$ l'est aussi; et réciproquement. Par conséquent, soit aucune des deux couleurs $D_{\alpha}$ et $D_{\beta}$ n'est une couleur de $X$, soit toutes les deux sont des couleurs de $X$.

Cette classification permet notamment de donner une version effective, dans le cas des variétés horosphériques de Fano, d'un résultat de V. Alexeev et $\mathrm{M}$. Brion [1] sur les variétés sphériques de Fano.

THÉORÈme 3.4. - Soit $G / H$ un espace homogène horosphérique de rang $n$. On note $a=\prod_{\alpha \in S \backslash I} a_{\alpha}$, et $V=(7(a+1))^{n 2^{n+1}}$. L'ensemble des classes d'isomorphisme des variétés de Fano qui sont des plongements de $G / H$ est fini et de cardinal inférieur à

$$
(n ! a V)^{\frac{n(n+1)}{2}} 2^{2^{n}(n ! a V)^{n+1}} .
$$

La borne obtenue est sans doute loin d'être optimale. En effet, les majorations faites dans la preuve sont en général assez grossières.

Le reste de cette section est consacré à la démonstration du théorème 3.4.

On appelle automorphisme de $(N, \mathcal{D})$ tout automorphisme du réseau $N$ qui fixe chaque $\check{\alpha}_{M}$. Grâce à la proposition suivante, il suffira de majorer le nombre de polytopes $G / H$-réflexifs à automorphisme de $(N, \mathcal{D})$ près pour démontrer le théorème 3.4 . 
Proposition 3.5. - Soit $\phi$ un automorphisme de $(N, \mathcal{D})$. On note aussi $\phi$ l'automorphisme de $N_{\mathbb{R}}$ induit par $\phi$. Soient $X$ et $X^{\prime}$ des plongements de $G / H$ d'éventails coloriés respectifs $\mathbb{F}$ et $\mathbb{F}^{\prime}$, tels que $\mathbb{F}^{\prime}=\phi(\mathbb{F})$. Alors les variétés $X$ et $X^{\prime}$ sont isomorphes.

Rappelons que les plongements $X$ et $X^{\prime}$ ne sont pas isomorphes si $\phi$ n'est pas trivial (théorème 2.5).

La preuve de cette proposition est fortement inspirée de [1].

Démonstration. - Notons $G^{\prime}$ la partie semi-simple de $G$. On définit alors $\tilde{G}=$ $G^{\prime} \times P / H$. C'est un groupe algébrique réductif et connexe. Rappelons que $P=N_{G}(H)$; ainsi $\tilde{G}$ agit sur $G / H$ par $(g, p H) . x H=g x p H$.

De plus, $G / H$ est homogène sous l'action de $\tilde{G}, G / H \simeq \tilde{G} / \tilde{H}$, et $\tilde{H}$ est un sous-groupe horosphérique de $\tilde{G}$. En effet, le radical unipotent $\tilde{U}$ du sous-groupe de Borel $\tilde{B}=\left(B \cap G^{\prime}\right) \times P / H$ est $U \times\{1\}$ et

$$
\tilde{H}=\{(g, p H) \in \tilde{G} \mid g p \in H\} \supset \tilde{U} .
$$

On vérifie aussi que $\tilde{P}:=N_{\tilde{G}}(\tilde{H})=\left(P \cap G^{\prime}\right) \times P / H$. Puis on voit que le réseau des caractères de $\tilde{P}$ dont la restriction à $\tilde{H}$ est triviale est le groupe $\left\{\left(\chi, \chi^{-1}\right), \chi \in M\right\}$ isomorphe à $M$. De plus, les couleurs associées à $\tilde{G} / \tilde{H}$ sont les mêmes que celles associées à $G / H$ avec les mêmes images dans $M$.

Ainsi les plongements de $G / H$ sont les mêmes que ceux de $\tilde{G} / \tilde{H}$. Donc $X$ et $X^{\prime}$ sont des plongements de $\tilde{G} / \tilde{H}$ d'éventails coloriés respectifs $\mathbb{F}$ et $\mathbb{F}^{\prime}$.

Comme $P / H$ est isomorphe au tore dual de $M, \phi$ induit un automorphisme de $P / H$, qu'on notera encore $\phi$. On peut alors définir $X^{\prime \prime}$ comme étant la variété $X$ sur laquelle $\tilde{G}$ agit par $(g, p H)_{\phi} \cdot x=g x \phi^{-1}(p H)$; c'est un plongement de $\tilde{G} / \tilde{H}$. Il suffit alors de montrer que son éventail colorié est $\mathbb{F}^{\prime}$ : on aura ainsi des isomorphismes de variétés $X^{\prime} \simeq X^{\prime \prime} \simeq X$.

Notons $\phi^{*}: M \longrightarrow M$ l'automorphisme dual de $\phi$. Comme $\phi$ fixe chaque couleur, $\phi^{*}(\chi)$ et $\chi$ ont alors la même restriction à $B \cap G^{\prime}$, pour tout $\chi \in M$.

Soit $f \in \mathbb{C}(\tilde{G} / \tilde{H})^{(\tilde{B})}$ de poids $\chi$; autrement dit, pour tous $(b, p H) \in \tilde{B}$ et $x \in \tilde{G} / \tilde{H}$, on a $f(b x p H)=\chi^{-1}(b) \chi(p) f(x)$.

Si l'action de $\tilde{G}$ est tordue par $\phi$, on a alors

$$
f\left((b, p H)_{\phi} \cdot x\right)=\chi^{-1}(b) \chi\left(\phi^{-1}(p H)\right) f(x) .
$$

Or $\chi\left(\phi^{-1}(p H)\right)=\phi^{*-1}(\chi)(p)$ par définition de $\phi^{*}$ et $\chi^{-1}(b)=\phi^{*-1}\left(\chi^{-1}\right)(b)$ car $\phi$ fixe chaque couleur. Donc $f$ est de poids $\phi^{*-1}(\chi)$ (lorsque l'action de $\tilde{G}$ est tordue par $\phi$ ).

Par conséquent, pour tout diviseur $\tilde{B}$-stable $D$ de $X$, en notant $D^{\prime \prime}$ le même diviseur dans $X^{\prime \prime}$, on a $\sigma\left(D^{\prime \prime}\right)=\phi(\sigma(D))$. De la façon dont l'éventail colorié d'un plongement est construit à partir des images par $\sigma$ des diviseurs $B$-stables, on conclut que $X^{\prime \prime}$ a pour éventail colorié $\mathbb{F}^{\prime}$. 
EXEMPle 3.5. - On sait déjà que le nombre de variétés toriques de Fano de dimension 2 est 16, dont 5 seulement sont lisses.

En rang 2 , il y a par exemple 135 polytopes $\left(S L_{2} \times \mathbb{C}^{*}\right) / U$-réflexifs, dont 16 correspondent à un plongement lisse et 398 polytopes $S L_{3} / U$-réflexifs, dont 27 correspondent à un plongement lisse. La liste complète de tous ces polytopes est donnée dans [22, ch.6].

Dans le cas torique, le théorème 3.4 dit que le nombre de polytopes réflexifs, à automorphisme de $\mathbb{Z}^{n}$ près, est fini. Ce résultat a été démontré en premier par A. Borisov et L. Borisov [4]. Mais une autre preuve (plus souvent utilisée) consiste à appliquer un résultat de D. Hensley [15] afin de majorer le volume des polytopes réflexifs.

Dans notre cas, on utilise une généralisation du théorème de D. Hensley due à J. Lagarias et G. Ziegler. On note $\stackrel{\circ}{Q}$ l'intérieur de $Q$.

THÉORÈme 3.6 ([18]). — Soient $n$, a et $k$ des entiers strictement positifs. Soit $Q \subset \mathbb{R}^{n}$ un polytope convexe à sommets dans $\mathbb{Z}^{n}$ tel que $\sharp\left(\stackrel{o}{Q} \cap a \mathbb{Z}^{n}\right)=k$. Alors le volume de $Q$ est majoré par $k a^{n}(7(k a+1))^{n 2^{n+1}}$.

Démonstration du théorème 3.4. - Commençons par remarquer qu'un polytope $G / H$-réflexif $Q$ est à sommets dans $\frac{1}{a} N$ et que $\stackrel{o}{Q} \cap N=\{0\}$. En effet, s'il existe $u$ dans $(\stackrel{o}{Q} \cap N) \backslash\{0\}$, alors pour tout $v \in Q^{*},\langle v, u\rangle>-1$. Or il existe un sommet $v$ de $Q^{*}$ tel que $\langle v, u\rangle<0$, ce qui contredit le fait que $\langle v, u\rangle$ est entier.

Par conséquent, le théorème précedent nous dit que tout polytope $G / H$ réflexif a un volume majoré par $V=(7(a+1))^{n 2^{n+1}}$.

Si $G$ est semi-simple, les $\frac{\check{\alpha}_{M}}{a_{\alpha}}$ pour $\alpha \in S \backslash I$ forment une famille génératrice de $N_{\mathbb{R}}$. Soient $\alpha_{1}, \ldots, \alpha_{n} \in S \backslash I$ tels que $\left(\check{\alpha}_{1 M}, \ldots, \check{\alpha}_{n M}\right)$ soit une base de $N_{\mathbb{R}}$. Soit $u \in Q$; pour tout $i \in\{1, \ldots, n\}$, le simplexe de sommets 0 , $u$, et $\frac{\check{\alpha}_{j M}}{\alpha_{j}}, j \neq i$ est strictement inclus dans $Q$, donc son volume est strictement inférieur à $V$. Par conséquent, $u$ est dans l'intérieur du parallélépipède de sommets $\pm n ! a V \check{\alpha}_{1 M}, \ldots, \pm n ! a V \check{\alpha}_{n M}$. Ce dernier contient au maximum $2^{n}(n ! a V)^{n+1}$ points de $N$, donc en majorant grossièrement, on obtient que le nombre de polytopes $G / H$-réflexifs est, dans ce cas, inférieur à $2^{2^{n}(n ! a V)^{n+1}}$.

Dans le cas général où $G$ est réductif, notons $N_{\mathbb{R}}^{1}$ le sous-espace vectoriel de $N_{\mathbb{R}}$ engendré par les $\check{\alpha}_{M}$. Soit $\left(\check{\alpha}_{1 M}, \ldots, \check{\alpha}_{l M}\right)$ une base de $N_{\mathbb{R}}^{1} ;$ pour tout polytope $G / H$-réflexif $Q$, il existe $f_{1}, \ldots, f_{n-l} \in Q \cap N$ tels que $\left.\left\{\check{\alpha}_{1 M}, \ldots, \check{\alpha}_{l M}\right)\right\} \cup$ $\left\{f_{1}, \ldots, f_{n-l}\right\}$ forme une base de $N_{\mathbb{R}}$. Montrons qu'il existe un ensemble fini fixé d'éléments de $N_{\mathbb{R}}$, tel qu'on puisse toujours se ramener, quitte à appliquer un automorphisme de $(N, \mathcal{D})$, au cas où les $f_{i}$ font partie de cet ensemble.

Soit $\left(e_{1}, \ldots, e_{n}\right)$ une base fixée de $N$ telle que $\left(e_{1}, \ldots, e_{l}\right)$ soit une base de $N^{1}=N_{\mathbb{R}}^{1} \cap N$. Dans cette base, un automorphisme de $(N, \mathcal{D})$ s'écrit alors 
matriciellement sous la forme $\mathcal{A}=\left(\begin{array}{cc}I_{l} & B \\ 0 & C\end{array}\right)$, où $I_{l}$ est l'identité dans $N^{1}$, $B \in M_{l, n-l}(\mathbb{Z})$ et $C \in G L_{n-l}(\mathbb{Z})$. Soit $\mathcal{F}$ la matrice formée des vecteurs colonnes $f_{1}, \ldots, f_{n-l}$ dans la base $\left(e_{1}, \ldots, e_{n}\right)$. Alors, il existe un automorphisme $\mathcal{A}$ de $(N, \mathcal{D})$ tel que $\mathcal{G}=\mathcal{A F}$ de la forme suivante (on note $\left(g_{i j}\right)_{1 \leq i \leq n, 1 \leq j \leq n-l}$ ses coefficients)

$$
\mathcal{G}=\left(\begin{array}{cccc}
g_{11} & & \cdots & g_{1, n-l} \\
\vdots & & & \vdots \\
g_{l+1,1} & & & \vdots \\
0 & \ddots & & \vdots \\
\vdots & \ddots & \ddots & \vdots \\
0 & \cdots & 0 & g_{n, n-l}
\end{array}\right)
$$

et telle que pour tout $1 \leq j \leq n-l$ et $1 \leq i \leq j-1$,

$$
0 \leq g_{i j}<\left|g_{j+l, j}\right| \text {. }
$$

De plus, le produit des $g_{j+l, j}$ est majoré par $n ! a V$, donc le nombre de telles matrices $\mathcal{G}$ est inférieur à $(n ! a V)^{\frac{n(n+1)}{2}}$.

On en déduit alors que le nombre de polytopes $G / H$-réflexifs est inférieur à

$$
(n ! a V)^{\frac{n(n+1)}{2}} 2^{2^{n}(n ! a V)^{n+1}} .
$$

Remarque 3.7. - Ce résultat peut se généraliser de la manière suivante : soit $\kappa$ un entier strictement positif; le nombre de plongements $X$ de $G / H$, tels que $-\kappa K_{X}$ soit de Cartier et ample, est fini. Il suffit de remarquer que le polytope $\mathbb{Q}-G / H$-réflexif $Q$ associé à $X$ a son dual à sommets dans $\frac{1}{\kappa} M$. On en déduit alors que $\frac{1}{\kappa} Q$ est à sommets dans $\frac{1}{a \kappa} N$, et son intérieur ne contient que 0 comme point entier. Ainsi le volume de $Q$ est majoré par $\kappa^{n}(7(a \kappa+1))^{n 2^{n+1}}$, et la suite de la démonstration reste inchangée.

\section{Majoration du degré et du nombre de Picard}

Pour majorer le degré et le nombre de Picard des variétés horosphériques de Fano, on se donne un espace homogène horosphérique $G / H$ et on étudie le degré et le nombre de Picard de ses plongements lisses. 
4.1. Majoration du degré. - Pour avoir plus de détails sur le degré des variétés de Fano, on se référera à [12] où le degré est majoré dans le cas des variétés toriques lisses, et aussi à [11] où on trouve en particulier des variétés toriques de Fano de grand degré (prop.5.22).

DÉfinition 4.1. - Soit $X$ une variété de Fano de dimension $d$. On appelle degré de $X$, le nombre d'intersection $\left(-K_{X}\right)^{d}$. Le théorème de Riemann-Roch et le théorème d'annulation de Serre impliquent que la dimension de $\Gamma\left(X,-k K_{X}\right)$ est équivalente, lorsque l'entier $k$ tend vers l'infini, à $\frac{k^{d}}{d !}\left(-K_{X}\right)^{d}$.

Le résultat suivant (cas particulier de [7, th.4.1]) se démontre en utilisant la remarque 3.2 et la formule des caractères de Weyl.

Proposition 4.2. - Soit $X$ un plongement de Fano de G/H. Alors

$$
\left(-K_{X}\right)^{d}=d ! \int_{Q^{*}} \prod_{\alpha \in R^{+} \backslash R_{I}^{+}} \frac{\left\langle 2 \rho^{P}+\chi, \check{\alpha}\right\rangle}{\left\langle\rho^{B}, \check{\alpha}\right\rangle} d \chi,
$$

où la mesure dans l'intégrale est celle pour laquelle le domaine fondamental de $M$ est de volume 1 .

Dans le cas torique, on a $\left(-K_{X}\right)^{d}=d ! \operatorname{vol}\left(Q^{*}\right)$ [21, cor 2.23]. Le théorème 3.6 permet alors de majorer le degré de $X$ de façon immédiate, mais cette borne est doublement exponentielle. O. Debarre donne une bien meilleure borne dans le cas où $X$ est lisse.

ThÉorème 4.1 ([12]). - Soit $X$ une variété torique lisse de Fano de dimension $n$ et de nombre de Picard $\rho$. Si $\rho>1$, alors $\left(-K_{X}\right)^{n} \leq n ! n^{\rho n}$.

Si $\rho=1$, on a bien sûr $X=\mathbb{P}^{n}$ et $\left(-K_{X}\right)^{n}=(n+1)^{n}$.

Dans le cas horosphérique, on obtient le résultat analogue énoncé dans l'introduction : le théorème 1.1 .

DÉFInITION 4.3. - Une variété normale est dite localement factorielle si tout diviseur de Weil est de Cartier.

A l'aide de la caractérisation des diviseurs de Cartier sur une variété sphérique [7, prop.3.1], on caractérise les plongements de Fano d'un espace homogène horosphérique $G / H$ qui sont localement factoriels, de la façon suivante : lorsque $X$ est de Fano.

Proposition 4.4. - Soit $G / H$ un espace homogène horosphérique. Soit $X$ un plongement de Fano de $G / H$. On note $Q$ le polytope $G / H$-réflexif associé.

Alors $X$ est localement factoriel si et seulement si pour toute face maximale $F$ de $Q$ on a :

(1) les seuls points de $F$ de la forme $\frac{\check{\alpha}_{M}}{a_{\alpha}}$ sont des sommets de $F$; 
(2) $F$ est un simplexe dont les sommets $e_{1}, \ldots, e_{n}$ vérifient :

(i) pour tout $i$, ou bien $e_{i} \in N$ ou bien il existe un unique $\alpha \in S \backslash I$ tel que $e_{i}=\frac{\check{\alpha}_{M}}{a_{\alpha}}$,

(ii) $\left(a_{1} e_{1}, \ldots, a_{n} e_{n}\right)$ est une base de $N$ où les $a_{i}$ sont définis par :

$$
a_{i}= \begin{cases}1 & \text { si } e_{i} \in N \\ a_{\alpha} & \text { si } e_{i}=\frac{\check{\alpha}_{M}}{a_{\alpha}} .\end{cases}
$$

La preuve de cette proposition est laissée au lecteur.

Lorsque $Q$ est le polytope $G / H$-réflexif associé à un plongement de Fano localement factoriel de $G / H$, on définira $a_{u}$ pour tout sommet $u$ de $Q$ comme dans la proposition.

Dans la suite, $G / H$ sera un espace homogène horosphérique fixé et $X$ un plongement de Fano localement factoriel de $G / H$.

On note $r$ l'entier strictement positif tel que le nombre de sommets de $Q$ soit égal à $n+r$.

Dans le cas torique, cet entier est le nombre de Picard. Dans le cas horosphérique, on peut exprimer le nombre de Picard de la façon suivante :

$$
\rho=m+\sharp(S \backslash I)-n=r+\sharp(S \backslash I)-\sharp\left(\mathcal{D}_{X}\right) .
$$

En effet, tout diviseur est linéairement équivalent à un diviseur de la forme $\sum_{i=1}^{m} b_{i} X_{i}+\sum_{\alpha \in S \backslash I} b_{\alpha} D_{\alpha}$ ( $b_{i}$ et $b_{\alpha}$ entiers) [7, Chap. 3.1], et les relations entre ces diviseurs sont les relations du type $\operatorname{div}(f)=0$ où $f \in \mathbb{C}(G / H)^{(B)}$. Pour la deuxième égalité, rappelons que $\sharp\left(\mathcal{D}_{X}\right)$ est le nombre de couleurs de $X$, c'est-àdire le nombre des points $\frac{\check{\alpha}_{M}}{a_{\alpha}}$ qui sont sur le bord de $Q$ (voir la remarque 3.3). Puisque $X$ est localement factoriel, les points de la forme $\frac{\check{\alpha}_{M}}{a_{\alpha}}$ sont tous des sommets de $Q$ et correspondent à une unique couleur. On en déduit que $\sharp\left(\mathcal{D}_{X}\right)$ est exactement le nombre de sommets de la forme $\frac{\check{\alpha}_{M}}{a_{\alpha}}$. Ainsi $m+\sharp\left(\mathcal{D}_{X}\right)=n+r$.

De plus, ces deux égalités restent vraies lorsque $X$ est seulement $\mathbb{Q}$-factoriel (voir la définition 4.6). Elles se démontrent par les mêmes arguments et avec la proposition 4.7 .

Exemple 4.2. - Si $r=1$, alors $Q$ est un simplexe : c'est l'enveloppe convexe de $e_{1}, \ldots, e_{n+1}$ et pour tout $i \in\{1, \ldots, n+1\},\left(a_{1} e_{1}, \ldots, a_{i-1} e_{i-1}, a_{i+1} e_{i+1}, \ldots\right.$, $\left.a_{n+1} e_{n+1}\right)$ est une base de $N$. Donc $a_{n+1} e_{n+1}=-a_{1} e_{1}-\cdots-a_{n} e_{n}$.

Par la suite on verra que le cas où $r=1$ est un cas un peu à part, comme dans le cas torique.

Une première étape pour démontrer le théorème 1.1 est de majorer le volume de $Q^{*}$. 
Proposition 4.5. - Soient $X$ un plongement de Fano localement factoriel de $G / H$ et $Q$ le polytope $G / H$-réflexif associé. On note

$$
C=n+\sum_{\alpha \in S \backslash I}\left(a_{\alpha}-1\right) .
$$

Si $\rho \geq 2$, alors

$$
\operatorname{vol}\left(Q^{*}\right) \leq\left(C^{r} \max _{\alpha \in S \backslash I} a_{\alpha}\right)^{n}
$$

et si $\rho=1$, on $a$

$$
\operatorname{vol}\left(Q^{*}\right) \leq\left((C+1) \max _{\alpha \in S \backslash I} a_{\alpha}\right)^{n} .
$$

On peut observer que $C$ ne dépend que de $G$ et $H$. On verra par la suite (lemme 4.8) que $C \leq d$.

La preuve de cette proposition est inspirée de la preuve du théorème 4.1 [12]. Elle consiste en deux lemmes.

Lemme 4.3. - Soit b un réel strictement positif. Si pour tout $u \in Q$ et pour tout $v \in Q^{*}$, on $a-1 \leq\langle v, u\rangle \leq b$, alors

$$
\operatorname{vol}\left(Q^{*}\right) \leq\left((b+1) \max _{\alpha \in S \backslash I} a_{\alpha}\right)^{n} .
$$

Démonstration. - Par définition du dual d'un polytope, on a $-\frac{1}{b} Q^{*} \subset Q^{*}$ : en effet si $v \in-\frac{1}{b} Q^{*}$, on a bien $\langle v, u\rangle \geq-1$ pour tout $u \in Q$ par hypothèse.

Raisonnons par l'absurde et supposons que $\operatorname{vol}\left(Q^{*}\right)>\left((b+1) \max _{\alpha \in S \backslash I} a_{\alpha}\right)^{n}$. Soient un réel $\eta \in] 0,1[$, et

$$
Q^{\prime}=\frac{1-\eta}{(b+1) \max _{\alpha \in S \backslash I} a_{\alpha}} Q^{*} .
$$

Par hypothèse, le volume de $Q^{\prime}$ est strictement supérieur à 1 pour $\eta$ assez petit. Donc, par le théorème de van der Corput [10], il existe deux éléments $q$ et $q^{\prime}$ de $Q$ tels que $q-q^{\prime}$ soit dans $M$. De plus $Q^{\prime}$ est convexe et $-\frac{1}{b} Q^{\prime} \subset Q^{\prime}$, donc

$$
\frac{q-q^{\prime}}{b+1}=\frac{q-b\left(-\frac{1}{b} q^{\prime}\right)}{b+1} \in Q^{\prime} \text {, et } q-q^{\prime} \in(b+1) Q^{\prime} \subset \frac{1}{\max _{\alpha \in S \backslash I} a_{\alpha}} Q^{*} .
$$

Il reste à montrer que 0 est le seul point de $M$ dans l'intérieur de $\frac{1}{\max _{\alpha \in S \backslash I} a_{\alpha}} Q^{*}$ pour obtenir la contradiction voulue.

Soit $v \in M$ non nul dans l'intérieur de $\frac{1}{\max _{\alpha \in S \backslash I} a_{\alpha}} Q^{*}$. Pour tout $u \in Q$, on a

$$
\langle v, u\rangle>-\frac{1}{\max _{\alpha \in S \backslash I} a_{\alpha}} .
$$

Or il existe un sommet $u$ de $Q$ tel que $\langle v, u\rangle$ soit strictement négatif, et ce sommet est dans $\frac{1}{a_{\alpha}} \mathbb{Z}$ pour un $\alpha \in S \backslash I$ donné. Ceci n'est pas possible, donc un tel $v$ n'existe pas. 
EXEMPLE 4.4. - Revenons à l'exemple 4.2 où $r=1$; on a pour tout $i \in$ $\{1, \ldots, n+1\}, a_{i}\left(1+\left\langle v_{j}, e_{i}\right\rangle\right)=\delta_{i j}\left(a_{1}+\cdots+a_{n+1}\right)$ où $v_{j}$ est le sommet de $Q^{*}$ associé à la face de $Q$ opposée à $e_{j}$, et $\delta_{i j}$ est le symbole de Kronecker. Dans ce cas, on peut prendre $b=a_{1}+\cdots+a_{n+1}-1$. Regardons ce que vaut $b$ selon les valeurs de $\rho$. Sachant que $\rho$ vaut 1 plus le nombre de racines simples $\alpha \in S \backslash I$ telles que $\frac{\check{\alpha}_{M}}{a_{\alpha}}$ ne soit pas un sommet de $Q$, quand $\rho>1$, la somme $a_{1}+\cdots+a_{n+1}$ peut être majorée par $C$. Par contre, si $\rho=1, a_{1}+\cdots+a_{n+1}$ vaut exactement $C+1$. On obtient dans les deux cas le résultat de la proposition 4.5 lorsque $r=1$.

Le lemme suivant généralise le résultat obtenu dans l'exemple ci-dessus.

LEMME 4.5. - Si $r \geq 2$, alors pour tout sommet $u$ de $Q$ et tout sommet $v$ de $Q^{*}$, on $a$

$$
0 \leq a_{u}(1+\langle v, u\rangle) \leq C^{r}
$$

Démonstration. - Soit $v$ un sommet de $Q^{*}$. On note $e_{1}, \ldots, e_{n}$ les sommets de la face de $Q$ associée à $v$. Alors $\left(a_{1} e_{1}, \ldots, a_{n} e_{n}\right)$ est une base de $N$, et $\left\langle v, e_{i}\right\rangle=-1$ pour tout $i \in\{1, \ldots, n\}$.

On note $0<b_{1}<\cdots<b_{k}, k \leq r$, les éléments de l'ensemble suivant :

$$
\left\{a_{u}(1+\langle v, u\rangle) \mid u \text { sommet de } Q \text { distinct des } e_{i}\right\} \text {. }
$$

On va alors montrer par récurrence sur $j$ que $b_{j}$ est majoré par $C^{j}$.

Soient $j \in\{1, \ldots, k\}$ et $u$ un sommet de $Q$ tel que $a_{u}(1+\langle v, u\rangle)=b_{j}$. Soit $E$ un sous-ensemble de $\{1, \ldots, n\}$ non vide, minimal, tel que $u$ et les $\left(e_{i}\right)_{i \in E}$ ne soient pas sur une face commune de $Q$. Un tel sous-ensemble existe car $u$ et $e_{1}, \ldots, e_{n}$ ne sont pas sur la même face. Quitte à changer l'ordre des $e_{i}$, supposons $E=\{1, \ldots, \epsilon\}$. Posons $w=a_{u} u+a_{1} e_{1}+\cdots+a_{\epsilon} e_{\epsilon}$, alors $w$ est un élément de $N$.

Il existe une face de $Q$ telle que $w$ soit dans le cône engendré par cette face. De plus, cette face n'est pas celle correspondant à $v$. Il existe donc $u_{1}, \ldots, u_{s}$, parmi les sommets de cette face, distincts de $e_{1}, \ldots, e_{n}$ (mais pas forcément distincts entre eux), et $e_{1}^{\prime}, \ldots, e_{t}^{\prime}$ parmi $e_{1}, \ldots, e_{n}$ (toujours pas forcément distincts entre eux), tels que

$$
w=\sum_{i=1}^{s} a_{u_{i}} u_{i}+\sum_{i=1}^{t} a_{i}^{\prime} e_{i}^{\prime} .
$$

Posons $a_{i}^{\prime}=a_{e_{i}^{\prime}}$ pour simplifier les notations.

La relation (4.5.1) n'est pas triviale, car $u$ et les $\left(e_{i}\right)_{i \in E}$ ne sont pas sur une face commune de $Q$. De plus, pour tout $i \in\{1, \ldots, t\}, e_{i}^{\prime}$ n'est pas dans 
$\left\{e_{1}, \ldots, e_{\epsilon}\right\}$. En effet, si par exemple $e_{t}^{\prime}=e_{\epsilon}$, alors la relation (4.5.1) induit la relation non triviale suivante :

$$
a_{u} u+a_{1} e_{1}+\cdots+a_{\epsilon-1} e_{\epsilon-1}=\sum_{i=1}^{s} a_{u_{i}} u_{i}+\sum_{i=1}^{t-1} a_{i}^{\prime} e_{i}^{\prime} .
$$

On en déduit alors que $u, e_{1}, \ldots, e_{\epsilon-1}$ ne sont pas sur une face commune de $Q$, ce qui contredit la minimalité de $E$.

On va utiliser la relation (4.5.1) pour majorer $b_{j}$ en fonction de $b_{j-1}$. Pour cela, on va d'abord établir une relation entre les entiers $a_{u}, a_{u_{i}}, a_{i}$ et $a_{i}^{\prime}$.

Le point

$$
\frac{1}{a_{u}+\sum_{i=1}^{\epsilon} a_{i}} w
$$

est le barycentre des points $u, e_{1}, \ldots, e_{\epsilon}$ affectés des coefficients (strictement positifs) respectifs $a_{u}, a_{1}, \ldots, a_{\epsilon}$. Comme $u$ et $e_{1}, \ldots, e_{\epsilon}$ sont des sommets de $Q$ qui ne font pas partie d'une même face, ce barycentre est dans l'intérieur de $Q$. Par conséquent,

$$
\frac{\sum_{i=1}^{s} a_{u_{i}}+\sum_{i=1}^{t} a_{i}^{\prime}}{a_{u}+\sum_{i=1}^{\epsilon} a_{i}}<1,
$$

autrement dit,

$$
a_{u}+\sum_{i=1}^{\epsilon} a_{i} \geq \sum_{i=1}^{s} a_{u_{i}}+\sum_{i=1}^{t} a_{i}^{\prime}+1 .
$$

La relation 4.5.1 nous donne alors

$$
b_{j}=a_{u}(1+\langle v, u\rangle)=\sum_{i=1}^{s} a_{u_{i}}\left(1+\left\langle v, u_{i}\right\rangle\right)+a_{u}+\sum_{i=1}^{\epsilon} a_{i}-\sum_{i=1}^{s} a_{u_{i}}-\sum_{i=1}^{t} a_{i}^{\prime} .
$$

Si $s=0$ alors $b_{j}=a_{u}+\sum_{i=1}^{\epsilon} a_{i}-\sum_{i=1}^{t} a_{i}^{\prime} \leq a_{u}+\sum_{i=1}^{\epsilon} a_{i}$.

Sinon, $b_{j} \geq \sum_{i=1}^{s} a_{u_{i}}\left(1+\left\langle v, u_{i}\right\rangle\right)+1$ et donc $a_{u_{i}}\left(1+\left\langle v, u_{i}\right\rangle\right)<b_{j}$ pour tout $i \in\{1, \ldots, s\}$; autrement dit, $a_{u_{i}}\left(1+\left\langle v, u_{i}\right\rangle\right) \leq b_{j-1}$. Ainsi,

$$
\begin{aligned}
b_{j} & \leq s b_{j-1}+a_{u}+\sum_{i=1}^{\epsilon} a_{i}-\sum_{i=1}^{s} a_{u_{i}}-\sum_{i=1}^{t} a_{i}^{\prime} \\
& \leq s b_{j-1}+a_{u}+\sum_{i=1}^{\epsilon} a_{i}-s .
\end{aligned}
$$

Or $s \leq \sum_{i=1}^{s} a_{u_{i}} \leq \sum_{i=1}^{s} a_{u_{i}}+\sum_{i=1}^{t} a_{i}^{\prime} \leq a_{u}+\sum_{i=1}^{\epsilon} a_{i}$, donc

$$
b_{j} \leq s\left(b_{j-1}-1\right)+a_{u}+\sum_{i=1}^{\epsilon} a_{i} \leq\left(a_{u}+\sum_{i=1}^{\epsilon} a_{i}\right) b_{j-1} .
$$

En particulier si $j=1$, alors $s=0$ et donc $b_{1} \leq a_{u}+\sum_{i=1}^{\epsilon} a_{i}$. 
Pour conclure, il suffit juste de remarquer que si $\epsilon=n$ on a alors $r=1$. Donc $\epsilon \leq n-1$ et

$$
a_{u}+\sum_{i=1}^{\epsilon} a_{i} \leq n+\sum_{\alpha \in S \backslash I}\left(a_{\alpha}-1\right)=C
$$

car les entiers $a_{u}$ et $a_{i}$ valent soit 1 soit $a_{\alpha}$.

La proposition 4.5 se déduit alors facilement de ces deux lemmes : le lemme 4.5 donne le réel $b$ à utiliser dans le lemme 4.3.

Pour montrer le théorème 1.1, il suffit maintenant d'une part de majorer le terme à l'intérieur de l'intégrale de la proposition 4.2, et d'autre part de donner une borne explicite pour $C$.

LEMME 4.6. - Si $r \geq 2$, alors pour tout $\alpha \in R^{+} \backslash R_{I}^{+}$et tout $\chi \in Q^{*}$,

$$
0 \leq \frac{\left\langle 2 \rho^{P}+\chi, \check{\alpha}\right\rangle}{\left\langle\rho^{B}, \check{\alpha}\right\rangle} \leq C^{r} \max _{\alpha \in S \backslash I} a_{\alpha} .
$$

Démonstration. - Il suffit de montrer le résultat pour tout sommet $\chi \operatorname{de} Q^{*}$.

Soit $\alpha \in S \backslash I$.

Si $\frac{\check{\alpha}_{M}}{a_{\alpha}}$ est un sommet de $Q$, alors le lemme 4.5 nous dit que

$$
\frac{\left\langle 2 \rho^{P}+\chi, \check{\alpha}\right\rangle}{\left\langle\rho^{B}, \check{\alpha}\right\rangle}=a_{\alpha}\left(1+\left\langle\chi, \frac{\check{\alpha}_{M}}{a_{\alpha}}\right\rangle\right) \leq C^{r} .
$$

Si $\frac{\check{\alpha}_{M}}{a_{\alpha}}$ n'est pas un sommet de $Q$, on obtient une majoration légèrement plus grande. Comme $\frac{\check{\alpha}_{M}}{a_{\alpha}} \in Q$, il existe des sommets $u_{i}$ de $Q$ et des réels positifs $\lambda_{i}$ tels que $\sum \lambda_{i} \leq 1$ et $\frac{\check{\alpha}_{M}}{a_{\alpha}}=\sum \lambda_{i} u_{i}$. D'après le lemme 4.5 on a pour tout $i$, $\left\langle\chi, u_{i}\right\rangle \leq C^{r}-1$ car $a_{u_{i}} \geq 1$. On en déduit alors que

$$
1+\left\langle\chi, \frac{\check{\alpha}_{M}}{a_{\alpha}}\right\rangle \leq C^{r} \text { et donc } \frac{\left\langle 2 \rho^{P}+\chi, \check{\alpha}\right\rangle}{\left\langle\rho^{B}, \check{\alpha}\right\rangle} \leq C^{r} a_{\alpha} .
$$

Soit maintenant $\alpha \in R^{+} \backslash R_{I}^{+}$. Ecrivons $\alpha=\beta_{1}+\cdots+\beta_{s}$ où $\beta_{1}, \ldots, \beta_{s}$ sont des racines simples. On a alors

$$
\frac{\left\langle 2 \rho^{P}+\chi, \check{\alpha}\right\rangle}{\left\langle\rho^{B}, \check{\alpha}\right\rangle}=\frac{\sum_{i=1}^{s}\left\langle 2 \rho^{P}+\chi, \check{\beta}_{i}\right\rangle}{\sum_{i=1}^{s} 1} \leq \frac{s C^{r} \max _{\alpha \in S \backslash I} a_{\alpha}}{s} .
$$

REMARQUE 4.7. - Lorsque $\rho=1$, d'une part $r=1$ et d'autre part $\frac{\check{\alpha}_{M}}{a_{\alpha}}$ est un sommet de $Q$ pour tout $\alpha \in S \backslash I$. On a alors le même résultat en remplaçant $C^{r} \max _{\alpha \in S \backslash I} a_{\alpha}$ par $C+1$. 
Lemme 4.8. - On $a$ :

$$
\sum_{\alpha \in S \backslash I}\left(a_{\alpha}-1\right) \leq \operatorname{dim}(G / P)=\sharp\left(R^{+} \backslash R_{I}^{+}\right) .
$$

En particulier $C \leq d=n+\sharp\left(R^{+} \backslash R_{I}^{+}\right)$.

Démonstration. - La preuve se fait en étudiant les différents cas, mais avant tout, réduisons le nombre de ces cas.

Étape 1. - Pour tout $\alpha \in S \backslash I, a_{\alpha}=\left\langle 2 \rho^{P}, \check{\alpha}\right\rangle=\left\langle 2 \rho^{B}, \check{\alpha}\right\rangle-\left\langle 2 \rho_{I}, \check{\alpha}\right\rangle$ où $2 \rho_{I}=$ $\sum_{\beta \in R_{I}^{+}} \beta$. Ainsi, comme $\left\langle 2 \rho^{B}, \check{\alpha}\right\rangle=2$, on a

$$
\sum_{\alpha \in S \backslash I}\left(a_{\alpha}-1\right)=\sharp(S \backslash I)-\sum_{\alpha \in S \backslash I} \sum_{\beta \in R_{I}^{+}}\langle\beta, \check{\alpha}\rangle .
$$

Le résultat à montrer est donc

$$
\sum_{\alpha \in S \backslash I} \sum_{\beta \in R_{I}^{+}}-\langle\beta, \check{\alpha}\rangle \leq \sharp\left(R^{+} \backslash R_{I}^{+}\right)-\sharp(S \backslash I) .
$$

Étape 2. - Soit $\Gamma$ le diagramme de Dynkin de $G$. Pour tout $J \subset S$, notons $\Gamma_{J}$ le sous-graphe de $\Gamma$ dont les sommets sont les éléments de $J$ et les arêtes sont celles de $\Gamma$ qui relient deux éléments de $J$. Montrons qu'il suffit de montrer le résultat lorsque le diagramme de Dynkin $\Gamma_{I}$ est connexe. Supposons donc le résultat vrai dans ce cas. Soit $I=\bigsqcup_{j=1}^{t} I_{j}$ tel que $\Gamma_{I}=\bigsqcup_{j=1}^{t} \Gamma_{I_{j}}$ soit la décomposition de $\Gamma_{I}$ en composantes connexes. Notons $S_{j}$ l'ensemble des racines simples de $S$ liées à $I_{j}$ dans le diagramme de Dynkin $\Gamma_{S}$ de $G$ (c'està-dire les racines simples $\alpha$ telles qu'il existe une racine simple $\beta$ de $I_{j}$ avec $\langle\alpha, \breve{\beta}\rangle \neq 0)$. Alors

$$
\sum_{\alpha \in S \backslash I} \sum_{\beta \in R_{I}^{+}}-\langle\beta, \check{\alpha}\rangle=\sum_{j=1}^{t} \sum_{\beta \in R_{I_{j}}^{+}} \sum_{\alpha \in S_{j} \backslash I_{j}}-\langle\beta, \check{\alpha}\rangle
$$

ce qui, par hypothèse, est inférieur à $\sum_{j=1}^{t}\left(\sharp\left(R_{S_{j}}^{+} \backslash R_{I_{j}}^{+}\right)-\sharp\left(S_{j} \backslash I_{j}\right)\right)$.

Or $\sharp\left(R_{S_{j}}^{+} \backslash R_{I_{j}}^{+}\right)-\sharp\left(S_{j} \backslash I_{j}\right)$ est le nombre de racines positives non simples de $R_{S_{j}}^{+} \backslash R_{I_{j}}^{+}$. De plus, $R_{S_{j}}^{+} \cap R_{S_{k}}^{+}=S_{j} \cap S_{k}$ si $j \neq k$ car deux racines simples ne peuvent pas être toutes les deux liées à $I_{j}$ et $I_{k}$ (un diagramme de Dynkin ne contient pas de cycle). On a donc

$$
\sum_{j=1}^{t}\left(\sharp\left(R_{S_{j}}^{+} \backslash R_{I_{j}}^{+}\right)-\sharp\left(S_{j} \backslash I_{j}\right)\right) \leq \sharp\left(R^{+} \backslash R_{I}^{+}\right)-\sharp(S \backslash I) .
$$

Il suffit donc de montrer le résultat lorsque $\Gamma_{I}$ est connexe et $S$ est l'ensemble des racines simples liées à $I$. 
Étape 3. - On peut de plus supposer que $S \backslash I$ est un singleton. En effet, en regardant la réunion disjointe $\bigsqcup_{\alpha \in S \backslash I} R_{I \cup\{\alpha\}}^{+} \backslash R_{I}^{+}$incluse dans $R^{+} \backslash R_{I}^{+}$, on voit que

$$
\sum_{\alpha \in S \backslash I}\left(\sharp\left(R_{I \cup\{\alpha\}}^{+} \backslash R_{I}^{+}\right)-1\right) \leq \sharp\left(R^{+} \backslash R_{I}^{+}\right)-\sharp(S \backslash I) .
$$

Étape 4. - Il reste à calculer d'une part $-\sum_{\beta \in R_{I}^{+}}\langle\beta, \check{\alpha}\rangle$, et d'autre part $\sharp\left(R_{I \cup\{\alpha\}}^{+} \backslash R_{I}^{+}\right)-1$ dans les différents cas où $\Gamma_{I}$ est connexe et $S \backslash I$ est un singleton.

Le tableau ci-dessous résume le résultat des calculs en fonction du type des diagrammes de Dynkin $\Gamma_{I}$ et $\Gamma_{S}$. On peut remarquer qu'il y a deux façons différentes d'ajouter un sommet à un diagramme de type $A_{1}$ pour obtenir un diagramme de type $G_{2}$. Il y a aussi deux façons d'ajouter un sommet à un diagramme de type $A_{1}$ pour obtenir un diagramme de type $B_{2} ;$ l'une des façons est comptée dans le cas $A_{i}$ et $B_{i+1}$ et l'autre dans le cas $A_{i}$ et $C_{i+1}$ en identifiant $B_{2}$ et $C_{2}$.

\begin{tabular}{|c|c|c|}
\hline Types respectifs de $\Gamma_{I}$ et de $\Gamma_{S}$ & $-\sum_{\beta \in R_{I}^{+}}\langle\beta, \check{\alpha}\rangle$ & $\sharp\left(R_{I \cup\{\alpha\}}^{+} \backslash R_{I}^{+}\right)-1$ \\
\hline$A_{i}$ et $A_{i+1}$ pour $i \geq 0$ & $i$ & $i$ \\
\hline$A_{i}$ et $B_{i+1}$ pour $i \geq 1$ & $2 i$ & $\frac{i(i+3)}{2}$ \\
\hline$A_{i}$ et $C_{i+1}$ pour $i \geq 1$ & $i$ & $\frac{i(i+3)}{2}$ \\
\hline$A_{i}$ et $D_{i+1}$ pour $i \geq 3$ & $2(i-1)$ & $\frac{(i-1)(i+2)}{2}$ \\
\hline$A_{5}$ et $E_{6}$ & 9 & 20 \\
\hline$A_{6}$ et $E_{7}$ & 12 & 41 \\
\hline$A_{7}$ et $E_{8}$ & 15 & 91 \\
\hline$A_{1}$ et $G_{2}$ (flèche vers $\alpha$ ) & 3 & 4 \\
\hline$A_{1}$ et $G_{2}$ (flèche partant de $\alpha$ ) & 1 & 4 \\
\hline$B_{i}$ et $B_{i+1}$ pour $i \geq 2$ & $2 i-1$ & $2 i$ \\
\hline$B_{2}$ et $C_{3}$ & 4 & 4 \\
\hline$B_{3}$ et $F_{4}$ & 9 & 14 \\
\hline$C_{i}$ et $A_{i+1}$ pour $i \geq 3$ & $2 i$ & $2 i$ \\
\hline$C_{3}$ et $F_{4}$ & 6 & 14 \\
\hline$D_{i}$ et $D_{i+1}$ pour $i \geq 4$ & $2 i-2$ & $2 i-1$ \\
\hline$D_{5}$ et $E_{6}$ & 10 & 15 \\
\hline$D_{6}$ et $E_{7}$ & 15 & 32 \\
\hline$D_{7}$ et $E_{8}$ & 21 & 78 \\
\hline$E_{6}$ et $E_{7}$ & 16 & 26 \\
\hline$E_{7}$ et $E_{8}$ & 27 & 56 \\
\hline
\end{tabular}

BUlLETIN DE LA SOCiÉTÉ MATHÉMATIQUE DE FRANCE 
Voici les calculs effectués dans le premier cas, c'est-à-dire lorsque $\Gamma_{I}$ est de type $A_{i}$ et que $\Gamma_{S}$ est de type $A_{i+1}$. Notons $\alpha, \beta_{1}, \ldots, \beta_{i}$ les racines simples de $S$,

alors

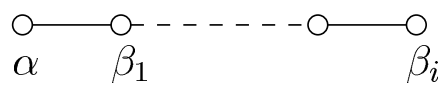

$$
\begin{aligned}
-\sum_{\beta \in R_{I}^{+}}\langle\beta, \check{\alpha}\rangle=-\sum_{1 \leq j \leq k \leq i}\left\langle\beta_{j}+\cdots+\beta_{k}, \check{\alpha}\right\rangle= & \\
& -\sum_{1 \leq k \leq i}\left\langle\beta_{1}+\cdots+\beta_{k}, \check{\alpha}\right\rangle=-\sum_{1 \leq k \leq i}-1=i .
\end{aligned}
$$

Puis $R_{I \cup\{\alpha\}}^{+} \backslash R_{I}^{+}=\left\{\alpha+\beta_{1}+\cdots+\beta_{k} \mid 1 \leq k \leq i\right\}$.

Les autres cas sont laissés au lecteur.

On peut remarquer que les configurations où il y a égalité sont celles qu'on retrouve lorsqu'on s'intéresse aux variétés horosphériques non toroïdales lisses (voir [22, déf.2.4]). Le résultat de ce lemme est alors optimal même dans le cas lisse.

On a maintenant tous les outils pour démontrer le théorème 1.1.

Démonstration. - Si $\rho>1$ alors les propositions 4.2 et 4.5 ainsi que les lemmes 4.6 et 4.8 nous permettent de dire que

$$
\left(-K_{X}\right)^{d} \leq d !\left(d^{r} \max _{\alpha \in S \backslash I} a_{\alpha}\right)^{n}\left(d^{r} \max _{\alpha \in S \backslash I} a_{\alpha}\right)^{d-n}=d ! d^{r d}\left(\max _{\alpha \in S \backslash I} a_{\alpha}\right)^{d} .
$$

On a évidemment $\max _{\alpha \in S \backslash I} a_{\alpha} \leq C \leq d$ dès que $n \geq 1$. D'autre part, si $n=0$ on a $X=G / P$ et $\rho=\sharp(S \backslash I)>1$; donc on a aussi $\max _{\alpha \in S \backslash I} a_{\alpha} \leq C$ puisque $a_{\alpha} \geq 2$ pour tout $\alpha \in S \backslash I$.

Si $\rho \geq r+1$ alors $\left(-K_{X}\right)^{d} \leq d ! d^{d \rho}$.

Si $\rho=r>1, \frac{\check{\alpha}_{M}}{a_{\alpha}}$ est un sommet de $Q$ pour tout $\alpha \in S \backslash I$. La preuve du lemme 4.6 nous dit que

$$
\frac{\left\langle 2 \rho^{P}+\chi, \check{\alpha}\right\rangle}{\left\langle\rho^{B}, \check{\alpha}\right\rangle} \leq C^{r}
$$

et donc

$$
\left(-K_{X}\right)^{d} \leq d !\left(d^{r} \max _{\alpha \in S \backslash I} a_{\alpha}\right)^{n}\left(d^{r}\right)^{d-n}=d ! d^{r d}\left(\max _{\alpha \in S \backslash I} a_{\alpha}\right)^{n} \leq d ! d^{d \rho+n} .
$$

De même, si $\rho=1$ on a aussi $r=1$ et

$$
\begin{gathered}
\left(-K_{X}\right)^{d} \leq d !\left((d+1) \max _{\alpha \in S \backslash I} a_{\alpha}\right)^{n}((d+1))^{d-n}= \\
d !(d+1)^{d}\left(\max _{\alpha \in S \backslash I} a_{\alpha}\right)^{n} \leq d !(d+1)^{d+n} .
\end{gathered}
$$

TOME $136-2008-\mathrm{N}^{\mathrm{O}} 2$ 
4.2. Majoration du nombre de Picard. - On va majorer le nombre de Picard des variétés horosphériques $\mathbb{Q}$-factorielles.

DÉFInItion 4.6. - Une variété normale est dite $\mathbb{Q}$-factorielle si tout diviseur de Weil est $\mathbb{Q}$-Cartier.

On va formuler un critère analogue à celui donné dans la proposition 4.4, toujours en utilisant la caractérisation des diviseurs de Cartier sur une variété sphérique [7, prop.3.1] énoncée dans la proposition 3.2. La preuve est laissée au lecteur.

Proposition 4.7. - Soit $X$ un plongement de Fano de $G / H$. On note $Q$ le polytope $G / H$-réflexif associé. Alors $X$ est $\mathbb{Q}$-factoriel si et seulement si toute face $F$ de $Q$ est un simplexe, et tous les points de $F$ de la forme $\frac{\check{\alpha}_{M}}{a_{\alpha}}$ sont des sommets de $F$.

On va montrer un résultat analogue à celui obtenu par C. Casagrande dans le cas torique [9, th.1(i)].

ThÉORÈme 4.9. - Soit $X$ une variété horosphérique de Fano, $\mathbb{Q}$-factorielle, de rang $n$, de dimension $d$ et de nombre de Picard $\rho$. Alors

$$
\rho \leq 2 n+\sharp(S \backslash I) \leq n+d \leq 2 d .
$$

On en déduit alors facilement le théorème 1.2 énoncé dans l'introduction, en remarquant que $n+d=2 d$ si et seulement si $X$ est torique.

La démonstration qui suit est inspirée des preuves de C. Casagrande [9, th.3(i)] et de B. Nill [20, lem.5.5].

Démonstration. - Soit $v$ un sommet de $Q^{*}$.

Étape 1. - Notons $F_{v}$ la face de $Q$ associée à $v$, et $e_{1}, \ldots, e_{n}$ les sommets de cette face. Montrons que tout sommet entier $u$ (c'est-à-dire dans $N$ ) de $Q$ vérifiant $\langle v, u\rangle=0$ est adjacent à $F_{v}$, c'est-à-dire qu'il existe un indice $j$ tel que $e_{1}, \ldots, e_{j-1}, e_{j+1}, \ldots, e_{n}$ et $u$ soient les sommets d'une face $F_{j}$ de $Q$. On note $u^{j}$ le sommet $u$ de $Q$ vérifiant cette dernière condition.

Soit $\left(e_{1}^{*}, \ldots, e_{n}^{*}\right)$ la base duale de $\left(e_{1}, \ldots, e_{n}\right)$ dans $M_{\mathbb{R}}$. Soit $j \in\{1, \ldots, n\}$. Alors $\left\langle e_{j}^{*}, u^{j}\right\rangle \neq 0\left(\operatorname{sinon} u^{j}\right.$ est dans l'hyperplan engendré par les $\left.\left(e_{i}\right)_{i \neq j}\right)$ et on peut alors définir

$$
\gamma_{j}=\frac{-1-\left\langle v, u^{j}\right\rangle}{\left\langle e_{j}^{*}, u^{j}\right\rangle} .
$$

De plus, le sommet de $Q^{*}$ associé à $F_{j}$ est $v^{j}=v+\gamma_{j} e_{j}^{*}$. On en déduit alors que $\gamma_{j}>0, \operatorname{car}\left\langle v^{j}, e_{j}\right\rangle>-1$. 
Soit $u$ un sommet entier de $Q$ vérifiant $\langle v, u\rangle=0$; alors $\left\langle v^{j}, u\right\rangle=\gamma_{j}\left\langle e_{j}^{*}, u\right\rangle$ et donc

$$
u \notin F_{j} \Longleftrightarrow\left\langle e_{j}^{*}, u\right\rangle \geq 0 .
$$

Si $u \neq u^{j}$ pour tout $j \in\{1, \ldots, n\}$, alors $\left\langle e_{j}^{*}, u\right\rangle \geq 0$ pour tout $j \in\{1, \ldots, n\}$ et donc $u$ est dans le cône engendré par $e_{1}, \ldots, e_{n}$, ce qui n'est pas possible. Par conséquent, $u$ est l'un des $u^{j}$ et est donc adjacent à $F_{v}$.

Étape 2. - Le nombre de sommets de $Q$ tels que $\langle v, u\rangle=-1$ est $n$, et le nombre de sommets entiers de $Q$ tels que $\langle v, u\rangle=0$ est inférieur ou égal à $n$ par l'étape 1.

L'origine est dans $Q$, donc il existe des sommets $v_{1}, \ldots, v_{h}$ de $Q^{*}(h>0)$ et des entiers strictement positifs $m_{1}, \ldots, m_{h}$ tels que $m_{1} v_{1}+\cdots+v_{h} m_{h}=0$.

On note $I=\{1, \ldots, h\}, M=\sum_{i \in I} m_{i}$, et pour tout sommet entier $u$ de $Q$ on pose

$$
A(u)=\left\{i \in I \mid\left\langle v_{i}, u\right\rangle=-1\right\} \text { et } B(u)=\left\{i \in I \mid\left\langle v_{i}, u\right\rangle=0\right\} .
$$

On a alors, pour tout sommet entier $u$ de $Q$,

$$
\begin{aligned}
0 & =\sum_{i \in I} m_{i}\left\langle v_{i}, u\right\rangle \\
& =-\sum_{i \in A(u)} m_{i}+\sum_{i \notin A(u) \cup B(u)} m_{i}\left\langle v_{i}, u\right\rangle \\
& \geq-\sum_{i \in A(u)} m_{i}+\sum_{i \notin A(u) \cup B(u)} m_{i} \\
& =M-2 \sum_{i \in A(u)} m_{i}-\sum_{i \in B(u)} m_{i}
\end{aligned}
$$

et donc $M \leq 2 \sum_{i \in A(u)} m_{i}+\sum_{i \in B(u)} m_{i}$.

Sommons cette dernière inégalité sur tous les sommmets entiers $u$ de $Q$. On obtient alors, en notant $r^{\prime}$ le nombre de ces sommets :

$$
\begin{aligned}
r^{\prime} M & \leq \sum_{u} \sum_{i \in A(u)} 2 m_{i}+\sum_{u} \sum_{i \in B(u)} m_{i} \\
& =\sum_{i \in I} \sum_{u,\left\langle v_{i}, u\right\rangle=-1} 2 m_{i}+\sum_{i \in I} \sum_{u,\left\langle v_{i}, u\right\rangle=0} m_{i} \leq 3 n M
\end{aligned}
$$

donc le nombre de sommets entiers de $Q$ est inférieur ou égal à $3 n$. On en déduit alors facilement que le nombre $n+r$ de sommets de $Q$ est inférieur ou égal à $3 n+\sharp\left(\mathcal{D}_{X}\right)$. En utilisant l'équation (4.1.1), on a

$$
\rho=r+\sharp(S \backslash I)-\sharp\left(\mathcal{D}_{X}\right) \leq 2 n+\sharp(S \backslash I) .
$$

TOME $136-2008-\mathrm{N}^{\mathrm{O}} 2$ 
Corollaire 4.10. - Soit $X$ une variété horosphérique de Fano localement factorielle de dimension $d \geq 2$. Alors

$$
\left(-K_{X}\right)^{d} \leq d ! d^{3 d^{2}}
$$

Démonstration. - Lorsque $\rho \geq 2$ le degré est majoré par

$$
d ! d^{d(2 n+\sharp(S \backslash I))+n} \leq d ! d^{d(2 n+\sharp(S \backslash I)+n)},
$$

grâce aux théorèmes 1.1 et 4.9. Sachant que $n+\sharp(S \backslash I) \leq d(2.1 .1)$, on obtient facilement le résultat.

Lorsque $\rho=1$, on a $\left(-K_{X}\right)^{d} \leq d !(d+1)^{d+n} \leq d !(d+1)^{2 d} \leq d ! d^{3 d^{2}}$ dès que $d>1$.

REMARQUes 4.11 . - Le cas où $d=1$ et $\rho=1$ correspond à la droite projective; le degré vaut alors 2.

$\mathrm{Si}$ on fait le même raisonnement dans le cas torique (en combinant le théorème de Debarre et celui de Casagrande), on obtient une borne légèrement meilleure, $d ! d^{2 d^{2}}$ au lieu de $d ! d^{3 d^{2}}$.

\section{BIBLIOGRAPHIE}

[1] V. Alexeev \& M. Brion - «Boundedness of spherical Fano varieties », in The Fano Conference, Univ. Torino, Turin, 2004, p. 69-80.

[2] _ « Toric degenerations of spherical varieties », Selecta Math. (N.S.) 10 (2004), p. 453-478.

[3] V. V. BATYREV - «Dual polyhedra and mirror symmetry for Calabi-Yau hypersurfaces in toric varieties », J. Algebraic Geom. 3 (1994), p. 493-535.

[4] A. A. Borisov \& L. A. Borisov - «Singular toric Fano three-folds », Mat. Sb. 183 (1992), p. 134-141.

[5] N. Bourbaki - Groupes et algèbres de Lie, C.C.L.S., 1975, chapitres 7 et 8.

[6] M. BRION - «Sur l'image de l'application moment », in Séminaire d'algèbre Paul Dubreil et Marie-Paule Malliavin (Paris, 1986), Lecture Notes in Math., vol. 1296, Springer, 1987, p. 177-192.

[7] _ , « Groupe de Picard et nombres caractéristiques des variétés sphériques », Duke Math. J. 58 (1989), p. 397-424.

[8] _ _ Curves and divisors in spherical varieties », in Algebraic groups and Lie groups, Austral. Math. Soc. Lect. Ser., vol. 9, Cambridge Univ. Press, 1997, p. 21-34. 
[9] C. Casagrande - «The number of vertices of a Fano polytope », Ann. Inst. Fourier (Grenoble) 56 (2006), p. 121-130.

[10] J. VAN DER CORPUT - «Verallgemeinerung einer Mordellschen Beweismethode in der Geometrie der Zahlen II », Acta Arith. 2 (1936), p. 145-146.

[11] O. DEBARRE - Higher-dimensional algebraic geometry, Universitext, Springer, 2001.

[12] _ _ "Fano varieties », in Higher dimensional varieties and rational points (Budapest, 2001), Bolyai Soc. Math. Stud., vol. 12, Springer, 2003, p. $93-132$.

[13] W. Fulton - Introduction to toric varieties, Annals of Mathematics Studies, vol. 131, Princeton University Press, 1993, The William H. Roever Lectures in Geometry.

[14] R. HARTSHORne - Algebraic geometry, Springer, 1977, Graduate Texts in Mathematics, No. 52.

[15] D. Hensley - «Lattice vertex polytopes with interior lattice points », Pacific J. Math. 105 (1983), p. 183-191.

[16] J. E. Humphreys - Linear algebraic groups, Springer, 1975, Graduate Texts in Mathematics, No. 21.

[17] F. KNoP - « The Luna-Vust theory of spherical embeddings », in Proceedings of the Hyderabad Conference on Algebraic Groups (Hyderabad, 1989), Manoj Prakashan, 1991, p. 225-249.

[18] J. C. Lagarias \& G. M. Ziegler - «Bounds for lattice polytopes containing a fixed number of interior points in a sublattice », Canad. J. Math. 43 (1991), p. 1022-1035.

[19] D. Luna \& T. Vust - «Plongements d'espaces homogènes », Comment. Math. Helv. 58 (1983), p. 186-245.

[20] B. NiLL - «Gorenstein toric Fano varieties », Manuscripta Math. 116 (2005), p. 183-210.

[21] T. ODA - Convex bodies and algebraic geometry, Ergebnisse der Mathematik und ihrer Grenzgebiete (3), vol. 15, Springer, 1988, An introduction to the theory of toric varieties.

[22] B. PAsquier - «Variétés horosphériques de Fano », Thèse, Université Joseph-Fourier, Grenoble I, 2006, http://www-fourier.ujf-grenoble.

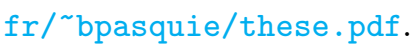

[23] _ « Smooth projective horospherical varieties with Picard number $1 »$, preprint arXiv: math/0703576, 2007.

[24] T. A. Springer - Linear algebraic groups, second éd., Progress in Mathematics, vol. 9, Birkhäuser, 1998. 
[25] D. Timashev - «Homogeneous spaces and equivariant embeddings », preprint arXiv:math.AG/0602228, 2006. 